\title{
The Study of RFID Authentication Protocols and Security of Some Popular RFID Tags
}

\author{
Hung-Yu Chien \\ Department of Information Management, National Chi Nan University, \\ Taiwan, R.O.C.
}

\section{Introduction}

A radio frequency identification (RFID) system consists of three components: radio frequency (RF) tags (or transponders), RF readers (or transceivers), and a backend server. Tag readers inquire tags of their contents by broadcasting an RF signal, without physical contact, at a rate of several hundred tags per second and from a range of several meters. The advancements of Silicon manufacturing also result in great cost reduction for RFID tags compared to barcodes, not to mention that the tags can carry more data and are more resistant to dust and twisting. Thanks to these excellent features, the world has seen many RFID systems already put to use by manufacturers and businesses of all kinds of goods for supply management and inventory control and such; in addition, many public facilities and parking lots have also brought in RFID systems to help them offer faster, easier and more user-friendly services. As a matter of fact, potential applications are everywhere [57]. Such features as great convenience, low cost, and wide applicability will soon make RFID systems the most pervasive microchips in history [57].

However, the wide distribution of RFID systems into modern society may very much likely get the security of both businesses and consumers exposed to threats and risks. For example, businesses may have malicious competitors on the market that collect unprotected RFIDs to gather information illegally, spread false tags to provide wrong information, or even launch denial of service (DOS) attacks against them. On the other hand, as a consumer, it is naturally preferred that the information of the purchase of RFID-tagged products be kept private from outsiders; however, a tag reader at a fixed location can read the content of an un-protected tag, tracing the RFID-tagged product or/and even identifying the person carrying the tagged product. Correlating data collected from multiple tag readers such as their locations and so on can also possibly be used to spy on an individual and track down his/her social interactions. Besides passive eavesdropping and tracking, a thief might use counterfeit tags to fool automated checkout or security systems into accepting wrong information like price, proof of presence or other information.

\section{RFID authentication protocols}

To protect the private information on the RFID tags, some special devices (such as a blocker tag [26]) can be used here to deter the reader from accessing the tags, or tag authenticates the reader before its access. An RFID authentication protocol is a cryptographic protocol that 
allows a reader and a tag to authenticate each other, and the protocol is especially suitable for cases where resource-limited RFID tags are involved. In fact, although there are highcost RFID tags like [25] available on the market that can support conventional symmetric key computations or even public key computations, the mainstream tags targeted at the majority of consumers are low-cost and can only support simple computations and very limited storage [50]. For example, for such tags as Gen $2[16,58]$ or iso 15693, conventional authentication protocols that require symmetric key computations or even public key computations are not applicable. Therefore, most of the efforts both the businesses concerned and the academic community have made so far are focused on the research and development of low-cost tags with higher security levels. Therefore, the topic of the next section is authentication protocols that are designed for low-cost RFIDs. Please also note that since well-designed conventional cryptographic protocols can be effectively implemented on resource-abundant backend servers and readers, it is usually assumed that the channels between backend servers and readers are secure; however, now that the focus is on RFID authentication protocols, this study has to assume that the channel between tags and readers is insecure. Figure 1 shows the components of an RFID system.

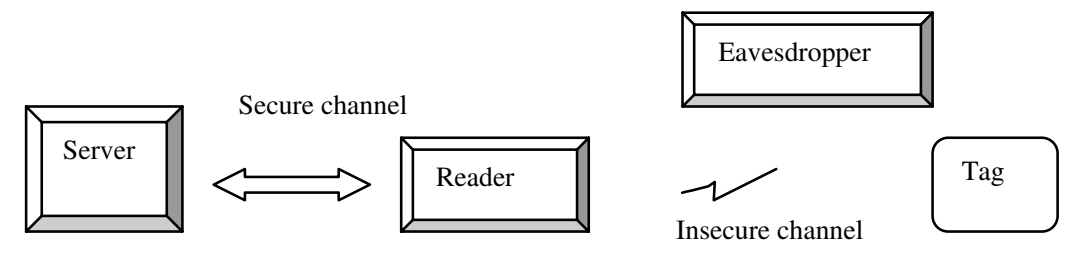

Fig. 1. Components of RFID systems

In addition, there are two special situations where the authentication of RFID tags is required to be done on extra conditions. To begin with, yoking proof protocols like $[4,7,23$, $24,48,53,60]$ require the proof of simultaneous presence of two (or more) tags, and RFID distance bounding protocols like $[5,39,56]$, on the other hand, not only authenticate the tags but also ensure that the authenticated tags are within a pre-assumed distance from the verifiers (the readers) so that the system is immune to message relay attacks like those brought up by [56]. In the following paragraphs, we shall briefly introduce yoking proof protocols and RFID distance bounding protocols. For detailed information, please refer to [4, 5, 7, 23, 24, 39, 48, 53, 56, 60].

\section{Yoking proof}

In 2004, Juels introduced an interesting RFID yoking proof protocol [23], which allows a verifier to prove the simultaneous presence of two tags in the communication range of a specific reader. Juels proposed several possible yoking proof protocol applications [23]. Let us take one example. Suppose a hard disk manufacturer wishes to ship each hard disk with its information leaflet. In such a case, each hard disk and each leaflet can be labeled with a different tag so that the yoking protocol can be applied to prove the simultaneous presence of the tagged products before shipping. In fact, the yoking proof protocol is a variant of the cryptographic authentication protocol, and it additionally requires the evidence of the simultaneous presence of two tags (or more tags). 


\section{RFID distance bounding protocols}

Due to the short communication range, an authenticated RFID tag is deemed to be in proximity by its verifier (for example, an RFID reader), and the security of many RFID applications depends on this proximity assumption. However, this belief of proximity could be maliciously manipulated and thus become misleading when relay attacks like [56] are launched. For example, the access control system of a building would allow the access only when an authenticated tag is in the proximity. However, a specific kind of relay attack named the mafia attack, introduced by Desmedt [14], could cheat the system where an attacker sets up a rogue tag (say $\hat{A}$ ) and a rogue reader (say $\hat{B}$ ) sitting between the real reader and the real tag, and $\hat{A}$ and $\hat{B}$ cooperatively relay the messages between the real tag and the real reader so that the real reader wrongly believes that the tag is in its proximity (but it is not). A distance bounding protocol is a cryptographic mechanism that can prevent relay attacks from working. It is executed by a tag $A$ and a reader $B$, and the $\operatorname{tag} A$ can convince the reader $B$ of $A^{\prime}$ s identity and $A^{\prime}$ s physical proximity to $B$.

\section{RFID authentication protocols}

An RFID authentication protocol provides mutual authentication between the reader and the tag, and should resist potential security threats and attacks like the replay attack, manin-the middle attack, etc. In addition to mutual authentication, anonymity and forward secrecy are also desirable properties for RFIDs. The point of ensuring the system's anonymity is to protect the privacy of the tags' identities such that un-authorized readers cannot identify or track a specific tag. Forward secrecy property, on the other hand, aims to protect the past communications where a tag is involved even if we assume that an attacker may have the power to compromise the tag some time later [50].

Just like tags of variant kinds currently available on the market, RFID authentication protocols can be quite different from one another, and the differences may come from the distinct resources required or the varied mechanisms adopted. Accordingly, we can classify these protocols and specify the features each kind has. Following the classification brought up by [52], for example, a protocol can be either a single-round design or a multi-round system. The former allows the reader and the tag to authenticate each other after a single round of operation of the protocol, while the latter has to run multiple rounds to do the job. Generally speaking, a single round protocol is more efficient than a multi-round protocol in terms of the number of interactions. Another classification, proposed by Chien [11], is based on the resources demanded by the protocols. This classification is very practical, because as we said earlier, on the market there are varieties of tags, of which most are resource-limited, and the resources required by these protocols can be very different. Under such circumstances, of course we will have a better view of the whole market if we classify the protocols and tags according to what kinds of resources are required. A third classification is based on the kind of cryptographic approach adopted, for the approach decides how well the protocol performs. Section 2.1 classifies the protocols as either single-round methods or multi-round methods, reviews the protocols and discusses the security properties. In Section 2.2, according to the required resources, we classify the protocols into four classes and introduce their corresponding applications. Finally, based on the cryptographic approaches, Section 2.3 classifies the protocols and discusses their performance. 


\subsection{RFID authentication protocols}

Some single-round protocols are introduced in Section 2.1.1 2.1.6, while multi-round protocols are introduced in Section 2.1.7. Even though tags' data and keys are stored in the backend server in most of the cases, we do not differentiate the role of backend sever and the reader to simplify the description in the following sections. The notations used are introduced as follows.

$r, r_{T}, r_{R}: l$-bit random numbers.

$I D_{T}, I D_{R}$ : the identity of tag $T$, the identity of reader $R$.

$k_{i}$ : the secret key shared between tag $T_{i}$ and the reader $R$.

$h(), g()$ : secure one-way hash function; $h(), g():\{0,1\}^{*} \rightarrow\{0,1\}^{l}$.

$C R C()$ : cyclic redundancy code.

$f()$ : a pseudo random number generator (PRNG function).

\subsubsection{Weis et al.'s schemes}

Weis et al. proposed a series of RFID authentication protocols [63, 64], and we review their hash-based access control protocol and the randomized access control.

Hash-based access control: Each hash-enabled tag $T_{i}$ in this design will have a portion of memory reserved for a temporary metaID $_{i}$ and will operate in either a locked state or an unlocked state. Initially, a tag owner stores the hash of a random key, $\operatorname{metaID}_{i} \leftarrow h\left(k_{i}\right)$, in the tag through either the RF channel or a physical contact to lock the tag. The owner also stores both the key and the metaID $D_{i}$ in a backend server. Upon receipt of a metaID $D_{i}$ value, the tag enters its locked state, and responds to all queries with only its metaID $D_{i}$ and offers no other functionality. To unlock a tag, the owner inquires the tag, looks up the appropriate key in the back-end database and finally transmits the key to the tag. The tag hashes the received

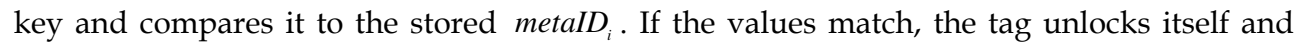
offers its full functionality to any nearby readers. The protocol is depicted in Figure 2.
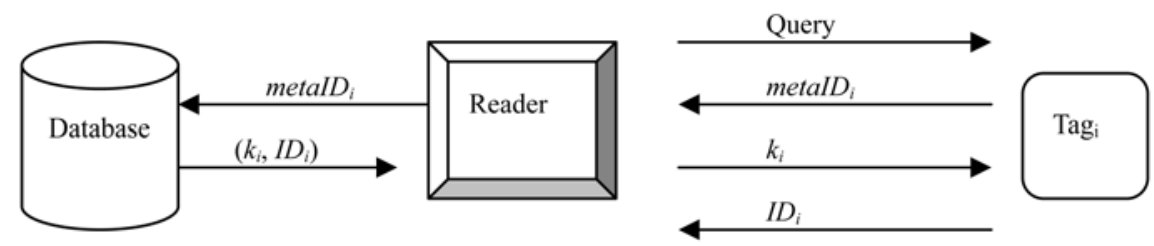

Fig. 2. Weis et al.'s Hash-based scheme: unlocking protocol

Randomized access control: In the previous scheme, a tag always responds with its metaID to the queries, which allows any party to track an individual. So, Weis et al. proposed their randomized access control schemes where a tag will not respond predictably to queries by unauthorized users, but must still identifiable by only legitimate readers. The randomized access control schemes require tags equipped with a random number generator, in addition to the one-way hash function. Upon receiving a query from the reader, a tag responds with the values $\left(r, h\left(I D_{i} \| r\right)\right)$, where $r$ is a randomly chosen number. A legitimate reader identifies one of its tags by performing a brute-force search of its known IDs, hashing each of them concatenated with $r$ until it finds a match. This mode is only feasible for owners of a relatively small number of tags. The protocol is depicted in Fig. 3. 

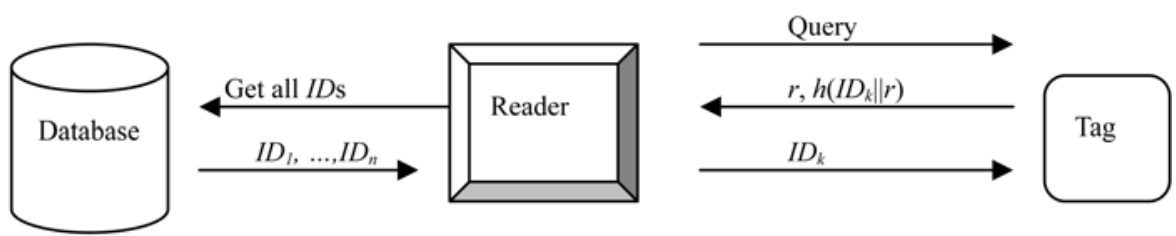

Fig. 3. Weis et al.'s randomized access control

Weakness of the hash-based scheme: In Figure 2, the reader broadcasts the tag's key in the forward channel. Since the signal in forward channel is strong enough for an adversary to monitor the transmission without being detected, this will allow an adversary easily eavesdrop the key and spoof a legal reader later.

Weaknesses of the random-access scheme: The Random-access scheme was designed to protect the metaID in the hash-based scheme to avoid individual tracking. However, it has poor scalability: it cannot support a large volume of tags because it has to perform the bruteforce search to find a matched ID. It also gives the adversary (who resides in the range of the backward channel) a very high probability to find the matched tag, since he also searches only a small database of possible IDs. What makes it worse: the legal reader will broadcast the matched ID in the forward channel. So, an adversary might record the eavesdropped data $\left(r, h\left(I D_{k} \| r\right)\right)$ and then easily spoofs the tags later.

\subsubsection{Ohkubo et al.'s scheme [43]}

The reader and each tag $T_{x}$ initially shares a distinct hash seed $s_{1_{-} x} . T_{x}$ updates $s_{i+1_{-} x}=h\left(s_{i_{-} x}\right)$ for $i \geq 1$ and responds with $a_{i_{-x}}=g\left(s_{i_{-} x}\right)$ in the $i$-th authentication, where $h() / g()$ are two different hash functions. The reader can follow the hashing chains to authenticate the tag. The protocol is depicted in Fig. 4.

This scheme provides only one-way authentication of the tag, but it owns the forward secrecy property; that is, even assuming a tag is compromised some day in the future, the past communications from the same tag can not be traced. However, Ohkubo et al.'s original version cannot resist the replay attack [1]- a simple replay of old message can cheat the reader into accepting a forged tag. The scheme has the poor scalability problem $[2,3]-$ the computational cost to identify a tag is $\mathrm{O}(\mathrm{nm})$, where $n$ is the number of potential tags and $m$ is the maximum length of the hash chain. Avoine et al. [1] discussed the techniques to conquer the replay attack, and Avoine et al. [1,2] also proposed their improvements to reduce the time complexity at the cost of extra memory.
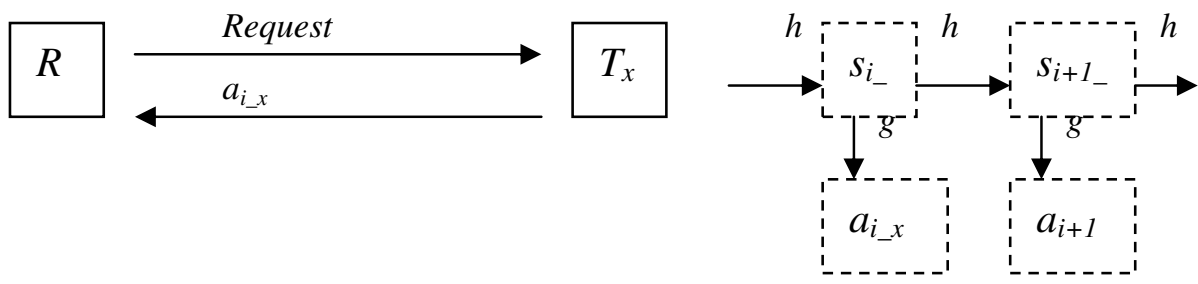

Fig. 4. Ohkubo et al.'s scheme 


\subsubsection{Karthikeyan-Nesterenko's scheme [28]}

Karthikeyanand and Nesterenko, based on simple XOR operation, $\oplus$, and matrix operation, designed an efficient tag identification and reader authentication scheme. Initially, two matrices $M_{1}$ and $M_{2}^{-1}$ are stored on each tag, and two matrices $M_{2}$ and $M_{1}^{-1}$ are stored on the reader, where all the matrices are of size $p \times p$, and $M_{1}^{-1}$ and $M_{2}^{-1}$ are the inverses of $M_{1}$ and $M_{2}$ respectively. The tag and the reader also store a key $K$ which is a vector of size $q$, where $q=r p$. That is, $K$ can be represented as $K=\left[K_{1}, K_{2}, \ldots, K_{\mathrm{r}}\right]$, where $K_{i}, i=1,2 \ldots, r$ are vectors of size $p$. As a slight abuse of notation, the notation $X=K M$, where $K$ is a vector of size $q$ and $M$ is a $p \times p$ matrix, denotes a component-wise multiplication of $K$ and $M$. That is, $X=\left[X_{1}, \ldots, X_{r}\right]=\left[K_{1} M, \ldots, K_{r} M\right]$.

When the reader inquires a tag, the tag computes $X=K M_{1}$, and sends back $X$ to the reader. The reader then forwards the message to the backend server, where the server will search its database to find a match. If it can find a match, then the tag is identified, and the server performs the following operations to authenticate itself to the tag and renew the key. The server first computes $Y=\left(K_{1} \oplus K_{2} \oplus \ldots \oplus K_{r}\right) M_{2}$, randomly selects a vector $X_{\text {new }}$ of size $q$, computes $K_{\text {new }}=X_{\text {new }} M_{1}^{-1}$ and $Z=K_{\text {new }} M_{2}$, and finally sends $(Y, Z)$ to the reader, which forwards $(Y, Z)$ to the tag. Upon receiving the response from the reader, the tag verifies whether the equation $Y M_{2}^{-1} \stackrel{?}{=}\left(K_{1} \oplus K_{2} \oplus \ldots \oplus K_{r}\right)$ holds; if so, the tag updates the key as $K_{\text {new }}=Z M_{2}^{-1}$. The scheme is depicted in Fig. 5.

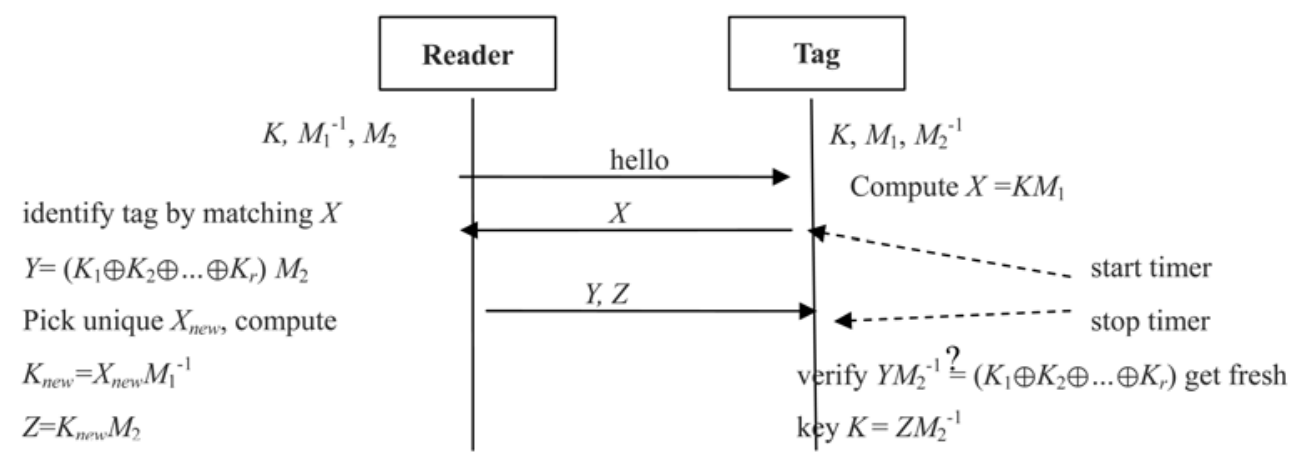

Fig. 5. Karthikeyan-Nesterenko's scheme

\section{Weaknesses of Karthikeyan-Nesterenko's scheme}

The scheme cannot resist the following attacks and threats- Denial of Services attack (DOS), replay attack and individual tracing.

In Karthikeyan-Nesterenko's scheme, the tag does not authenticate the received value $Z$ when updating the key. Therefore, an attacker can replace the transmitted $Z$ with an old one $\bar{Z}$ or any random value $Z^{*}$ without being noticed; Upon receiving a valid $Y$ and the fake $Z^{*}$, the tag will authenticate the $Y$ successfully and then will update the key as $K^{*}=M_{2}^{-1} \cdot Z^{*}$. So, the legitimate reader and the tag cannot authenticate each other any more since the key is wrongly updated.

If the attacker replaces the $Z$ with an old one $\bar{Z}$ (assuming $\bar{Y}$ and $\bar{Z}$ are previously sent in the $i$ th legal session) in the above mentioned attack, then the attacker can replay the $\bar{Y}$ in 
the next session to cheat the tag in wrongly accepting the request and access the tag accordingly. He can even record the transmitted data from several sessions, and then launches the above attack several times. This will allow the attacker to trace the tag. Therefore, the anonymity property is violated.

\subsubsection{Duc et al.'s scheme [15]}

Duc et al.'s scheme was designed for improving the security of EPCglobal Class-1 Generation-2 tag (which is called Gen-2 for short later). Initially, each tag and the backend server share the tag's EPC code (the identity of the tag), the tag's access PIN, and an initial key $K_{0}$ (this key will be updated after each successful authentication, and $K_{i}$ denotes the key after $i$ th authentication). The steps of $(i+1)$ th authentication are described as follows, where "Reader $\rightarrow$ tag: $M$ " denotes the reader sends the tag a message $M$.

1. Reader $\rightarrow$ tag: Query request.

2. Tag $\rightarrow$ reader $\rightarrow$ server: $M_{1}, r, C$.

The tag selects a random number $r$, computes $M_{1}=C R C(E P C \| r) \oplus K_{i} \quad$ and $C=C R C\left(M_{1} \oplus r\right)$, and sends back $\left(M_{1}, r, C\right)$ to the reader, where the reader will forward $\left(M_{1}, r, C\right)$ to the backend server.

3. Server $\rightarrow$ reader: the tag's info or "failure".

For each tuple $\left(E P C, K_{i}\right)$ in its database, the server verifies whether the equations $M_{1} \oplus K_{i} \stackrel{?}{=} C R C(E P C \| r)$ and $C \stackrel{?}{=} C R C\left(M_{1} \oplus r\right)$ hold. If it can find a match, then the tag is successfully identified and authenticated, and the server will forward the tag's information to the reader and proceed to the next step; otherwise, it stops the process with failure.

4. Server $\rightarrow$ Reader $\rightarrow$ tag: $M_{2}$

To authenticate itself to the tag and update the information on the tag, the server computes $M_{2}=C R C(E P C\|P I N\| r) \oplus K_{i}$ and sends $M_{2}$ to the tag through the reader. Upon receiving $M_{2}$, the tag uses its local values to verify the received $M_{2}$. If the verification succeeds, the tag will accept the "end session" command in the next step.

5. Reader $\rightarrow$ tag: "end session"

Reader $\rightarrow$ server: "end session".

- Upon receiving the "end session" command, both the server and the tag update their shared key as $K_{i+1}=f\left(K_{i}\right)$.

\section{The weaknesses}

Duc et al.'s scheme cannot resist the DOS attack against tags and readers, cannot detect the disguise of tags, and cannot provide forward secrecy.

(1) In the last step of Duc et al.'s scheme, the reader sends the "end session" commands to both the tag and the backend server to update the key. If one of the "end session" commands is intercepted, then the shared key between the tag and the server will be out of synchronization. Thus, the tag and the reader cannot authenticate each other any more. The DOS attack succeeds. (2) If it is the "end session" command to the server is intercepted, then the server will hold the old key; therefore, a counterfeit tag can replay the old data $\left(M_{1}, r, C\right)$ to disguise as a legitimate tag. So, the scheme fails to detect a disguised tag. (3) The scheme cannot provide forward secrecy. Suppose a tag is compromised, then the attacker would get the values $\left(E P C, P I N, K_{i}\right)$ of the tag ; So, from the eavesdropped data $\left(M_{1}, M_{2}, r\right)$ of the 


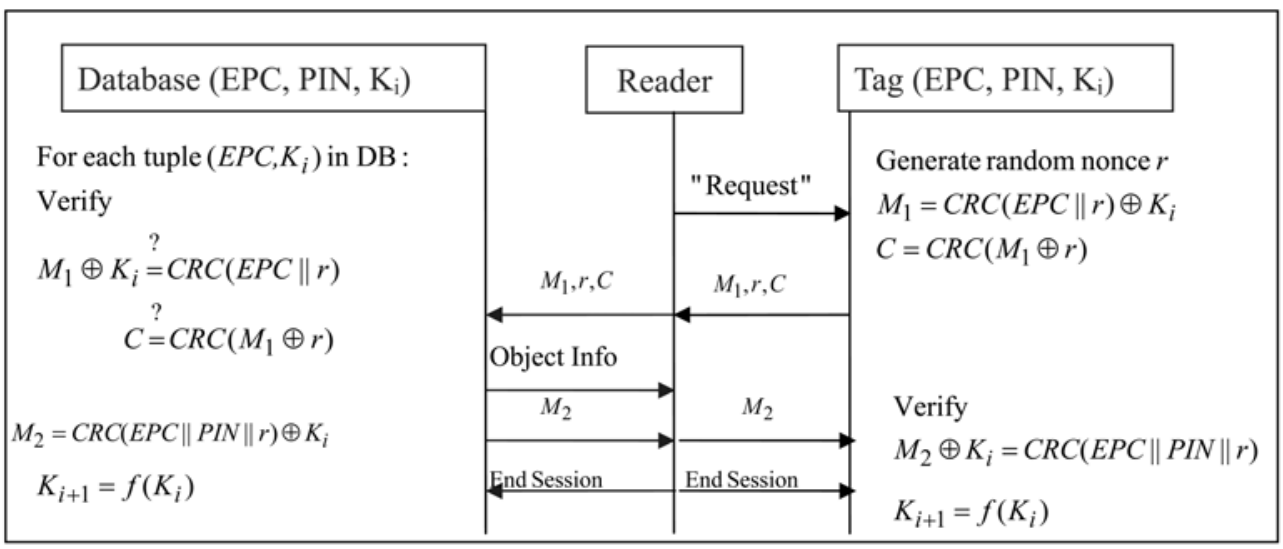

Fig. 6. Due et al. scheme

past communications, the attacker can verify whether a communication comes from the same tag by performing the following checking. For each eavesdropped communication $\left(M_{1}, \quad M_{2}, r\right)$, he computes $M_{1} \oplus M_{2}$ to derive the value $C R C(E P C \oplus r) \oplus$ $C R C(E P C\|P I N\| r)$, and then, using the compromised values $\left(E P C, P I N, K_{i}\right)$ and the eavesdropped $r$, he can do the same computation to verify whether it came from the same tag. So, the past communications of a compromised tag can be traced.

\subsubsection{Peris-Lopez et al.'s protocols [45-47]}

Peris-Lopez et al. proposed a series of ultra-lightweight RFID authentication protocols [4547] which were designed for very low-cost tags. Their schemes were very efficient: they require about 300 gates only and involve only simple bitwise operations. We review the LAMP protocol [45], which is one of Peris-Lopez et al.'s ultra-lightweight protocols.

LMAP involves only simple bitwise operations- bitwise XOR $(\oplus)$, bitwise $\operatorname{AND}(\wedge)$, bitwise OR $(\vee)$, and addition mod $2^{m}(+)$. The random number generator is only required on the reader. To protect the anonymity of tags, they adopt the technique of pseudonyms (IDSs), which is 96-bit length and is updated per successful authentication. Each tag shares an IDS and four keys (called K1,K2, K3, and K4, each with 96 bits) with readers, and they update the IDS and the keys after successful authentication. It needs 480 bits of rewritable memory and 96 bits for static identification number (ID).

The protocols consist of three stages- tag identification phase, mutual authentication phase, and pseudonym updating and key updating phase. In the following, $I D_{i}$ denotes the static identification of $\mathrm{Tag}_{\mathrm{i}}, I_{D} S_{i}^{n}$ denotes the pseudonym of $\mathrm{Tag}_{\mathrm{i}}$ at the $n$-th run, and $K 1_{i}^{n} / K 2_{i}^{n} / K 3_{i}^{n} / K 4_{i}^{n}$ denote the four keys of $\mathrm{Tag}_{\mathrm{i}}$ at the $n$-th run. LMAP is depicted in Fig. 7 . Tag identification: Initially, the reader sends "hello" to probe $\mathrm{Tag}_{\mathrm{i}}$, which responds with its current IDS ${ }_{i}^{n}$.

Mutual authentication phase: the reader uses $I D S_{i}^{n}$ to find the corresponding four keys in its database, via the help of the backend server. It then randomly selects two integers $n 1$ and 
$n 2$, and computes the values $A, B$, and $C$ (the calculation equations are specified in Fig. 7 ). From $A|| B|| C, \operatorname{Tag}_{i}$ first extracts $n 1$ from $A$, and then verifies the value of $B$. If the verification succeeds, then it extracts $n 2$ from $C$, and computes the response value $D$. Upon receiving $D$, the reader verify the data $D$ to authenticate the tag.

Pseudonym updating and key updating: After the reader and the tag authenticated each other, they update their local pseudonym and keys as specified in Fig. 7.

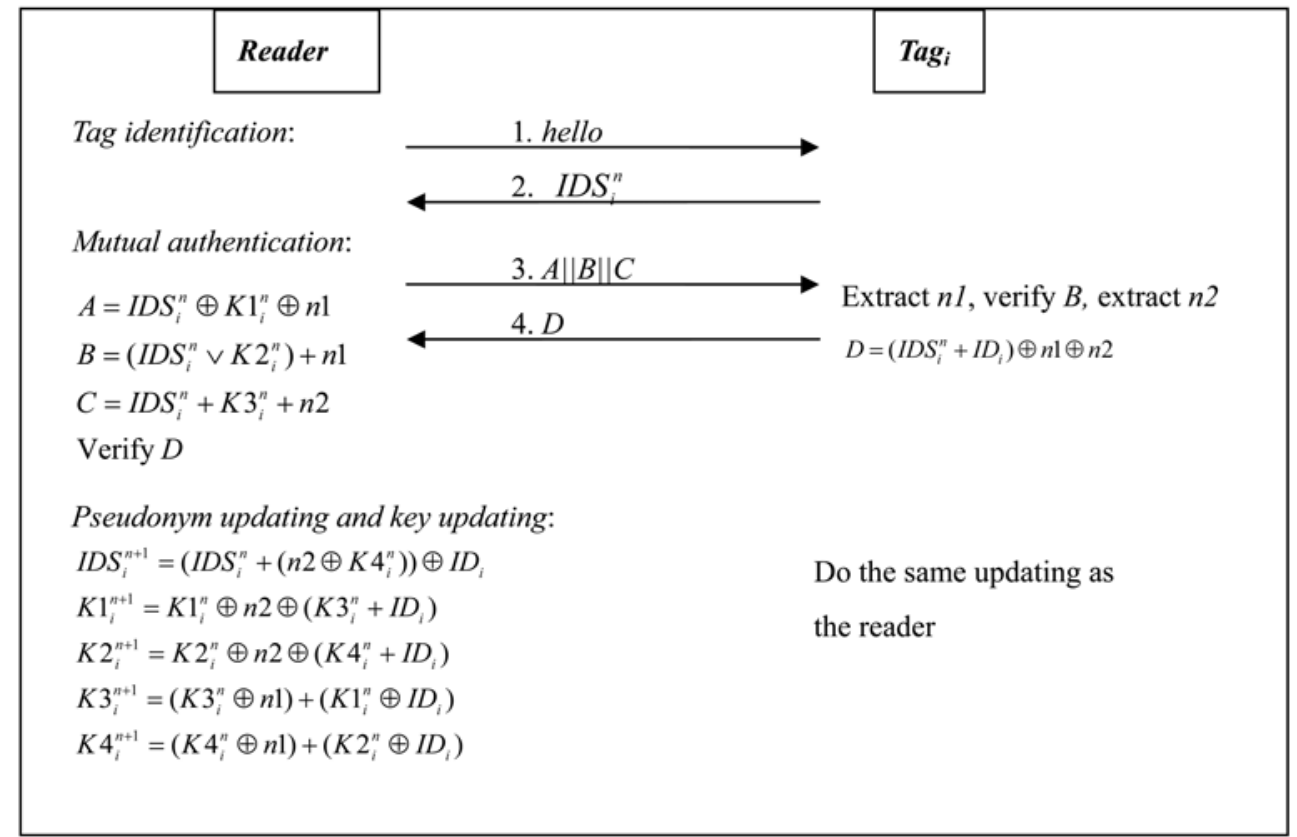

Fig. 7. LMAP

\section{The weaknesses}

The authentication of reader and tag in LMAP depends on the synchronization of pseudonym and keys. However, it is very easy to de-synchronize these values by intercepting the data in Step 4. In addition to the DOS attack, one can fully disclose the secrets of tags as follows.

We assume that an attacker can intercept, modify, and replay message between reader and tag in a reasonable time, and there is a completion message to indicate the completion of successful authentication. The attack scenario consists of five phases, but our attack is much more efficient than Li-Wang's work [34]. The whole scenario is depicted in Fig. 8.

In the attack scenario in Fig. 8, we omit the superscript $n$ and the subscript $i$ of pseudonym and of keys without causing ambiguity, since we are attacking the same tag within a successful session. In Phase 1, an attacker impersonates a reader and acquires the current IDS of a tag, and then the attacker (now impersonating the tag) uses the IDS to get a valid message $A|| B|| C$ from the reader in Phase 2.

In Phase 3 , the attacker iteratively inverts the $j$-th (for $1 \leq j \leq 96$ ) bit of $A$, modifies $B$, and sends $A_{j}^{\prime}\left\|B_{j}^{\prime}\right\| C$ to the tag. From the tag's response (which is either a message $D$ or an error 
message), the attacker can derive the $j$-th bit of $n 1$. After deriving the value of $n 1$, it further derives the values of $K 1$ and $K 2$ from $A, B, I D S$ and $n 1$. The detail of deriving the $j$-th bit is as follows. Let $A_{j}^{\prime}$ denotes the value by inverting the $j$-th bit of $A$. If the tag receives $A_{j}^{\prime}$, then it will derive $n_{1}$, which is equal to either $n 1+2^{j-1}$ or $n 1-2^{j-1}$, and each of the cases is with probability $1 / 2$. So, the attacker can assume $n_{1}{ }^{\prime}=n_{1}+2^{j-1}$, computes $B_{j}{ }^{\prime}=B+2^{i-1}$, and sends $A_{j}^{\prime}\left\|B_{j}^{\prime}\right\| C$ to the tag. After receiving $A_{j}^{\prime}\left\|B_{j}^{\prime}\right\| C$, the tag extracts $n_{1}^{\prime}$ from $A_{j}^{\prime}$, verifies $B_{j}^{\prime}$, and then responds with either a message $D_{j}$ or an error message. If a proper $D_{j}$ is returned, the attacker can conclude that $n_{1}{ }^{\prime}=n_{1}+2^{j-1}$ and $n 1[j]=0 \quad(n 1[j]$ denotes the $j$-th bit of $n 1)$; otherwise, it concludes that $n 1[j]=1$. With this technique, the attacker launches 96 runs to derive all the bits of $n_{1}$, and then solves the values of $K 1$ and $K 2$ accordingly. Now the rest is to derive the values of $n 2, K 3, K 4$ and $I D$.

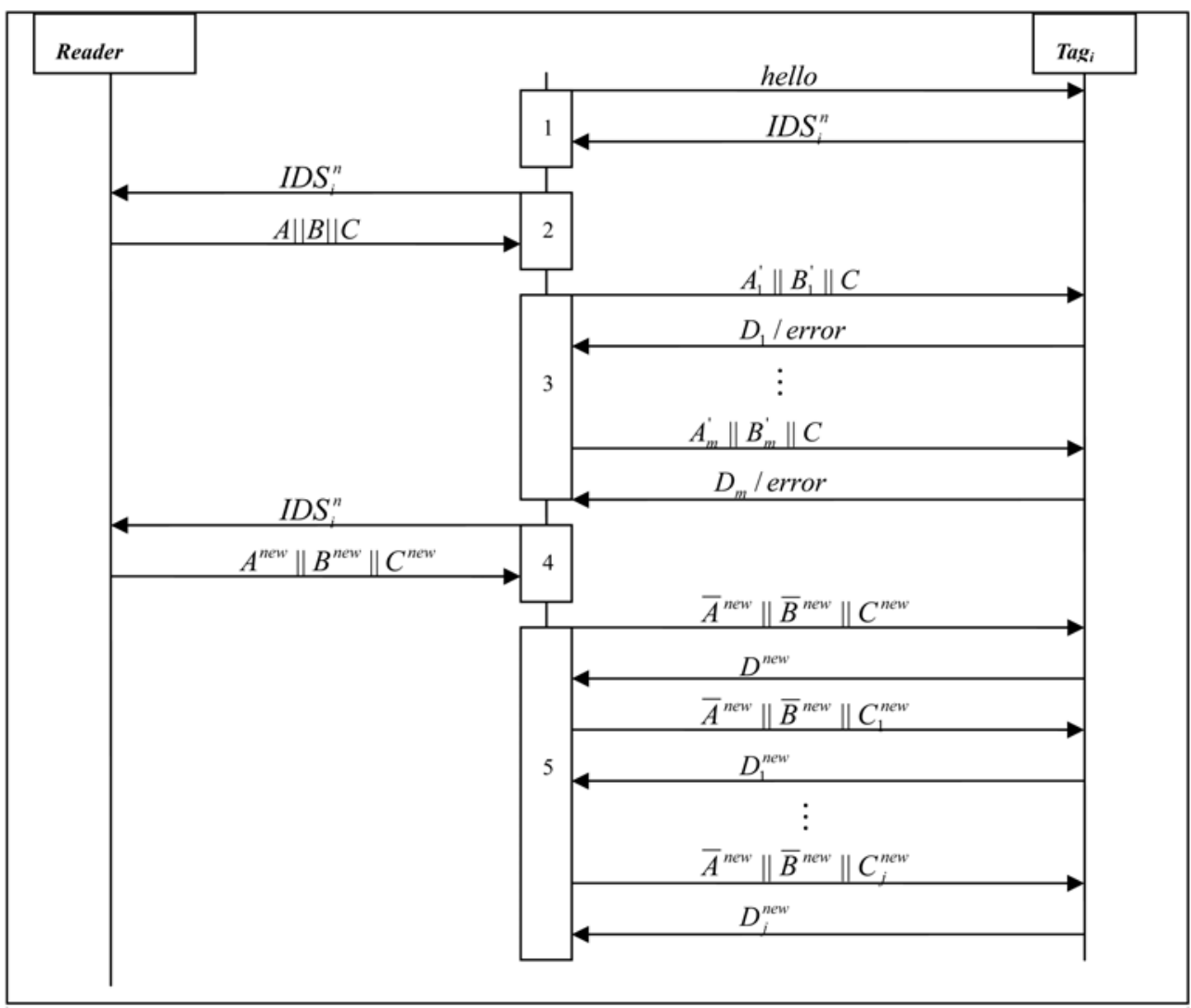

Fig. 8. Full-disclosure attack on LMAP

In Phase 4, the attacker impersonates the tag to the reader to get a new response $A^{\text {new }}\left\|B^{\text {new }}\right\| C^{\text {new }}$.

In phase 5, since the values of IDS, K1, and $K 2$ are already known, the attacker first sets $\overline{n 1}^{\text {new }}=0$ to have $\bar{A}^{\text {new }}=I D S \oplus K 1$ and $\bar{B}^{\text {new }}=I D S \vee K 2$, and sends $\bar{A}^{\text {new }}\left\|\bar{B}^{\text {new }}\right\| C^{\text {new }}$. So, the tag 
will respond with $D^{\text {new }}=(I D S+I D) \oplus n 2$. Next, the attacker sets $C_{1}^{\text {new }}=C^{\text {new }}+1$, and sends $\bar{A}^{\text {new }}\left\|\bar{B}^{\text {new }}\right\| C_{1}^{\text {new }}$ to the tag, which will extract $n 2+1$ and will respond with $D_{1}^{\text {new }}=(I D S+I D) \oplus(n 2+1)$. Now we have the equation $n 2 \oplus(n 2+1)=D^{\text {new }} \oplus D_{1}^{\text {new }}$. The possible values of $D^{\text {new }} \oplus D_{1}^{\text {new }}$ are summarized in Table 1. From Table 1, we can see that (1) if $D^{\text {new }} \oplus D_{1}^{\text {new }}$ has the form $0 \ldots 01$, then $n 2[1]=0$; (2) if $D^{\text {new }} \oplus D_{1}^{\text {new }}$ has $i+11$ s on the right and the rest are $0 \mathrm{~s}$, then $n 2$ has $i 1 \mathrm{~s}$ from the right and followed by a zero. So, two simple interactions with the tag, the attacker can determine $i+1(i \in[0,95])$ bits of $n 2$. Following that, the attacker sets $C_{i+2}^{\text {new }}=C^{\text {new }}+2^{i+1}$, and sends $\bar{A}^{\text {new }}\left\|\bar{B}^{\text {new }}\right\| C_{i+2}^{\text {new }}$. After getting the response $D_{i+2}^{\text {new }}$, the attacker computes $D^{\text {new }} \oplus D_{i+2}^{\text {new }}$ to determine the next few bits. It repeats this process until all the 96 bits of $n 2$ are solved. This phase takes 2 interactions in the best case and 96 interactions in the worst case. After deriving $n 2$, the attacker can further solve $K 3$ and $I D$ from the data $C$ and $D$. With two successive pseudonyms $I D S_{i}^{n}$ and $I D S_{i}^{n+1}$, the attacker further derives $K 4$.

\begin{tabular}{|l|l|}
\hline $\begin{array}{l}\text { If } n 2[1]=0 \text { (that is, } n 2 \text { has the form } \\
\operatorname{xxx} \ldots . . . \times 0)^{*}\end{array}$ & then $n 2 \oplus(n 2+1)=000 \ldots 01$ \\
\hline $\begin{array}{l}\text { If } n 2[1]=1 \text { and } n 2 \text { has the form } \mathrm{xxx} 01 \ldots 1 \\
\text { (that is, } n 2 \text { has } i \text { s from the right } \\
\text { followed by a } 0)\end{array}$ & $\begin{array}{l}\text { then } n 2 \oplus(n 2+1)=0 \ldots 01 \ldots 1 \text { (that is, } \\
n 2 \oplus(n 2+1) \text { has } i+11 \text { s on the right and the } \\
\text { rest are } 0 \mathrm{~s})\end{array}$ \\
\hline
\end{tabular}

${ }^{*} x$ denote the bit value is either 0 or 1 .

Table 1. The possible values of $D^{\text {new }} \oplus D_{1}^{\text {new }}$

For more details of weaknesses of Peris-Lopez et al.'s ultra-lightweight protocols [45-47], one can refer to [13, 33-35].

\subsubsection{Chien's SASI protocol [11]}

Chien's SASI was designed for very low-cost RFID tags. Each tag has a static identification (ID), and pre-shares a pseudonym (IDS) and two keys K1/K2 with the backend server. The length of each of ID/IDS/K1/K2 is 96 bits. To resist the possible de-synchronization attack, each tag actually keeps two entries of (IDS, K1, K2): one is for the old values and the other is for the potential next values. The protocol consist of three stages- tag identification phase, mutual authentication phase, and pseudonym updating and key updating phase. In each protocol instance, the reader may probe the tag twice or once in the tag identification phase, depending on the tag's IDS is found or not. The reader first sends "hello" message to the tag, and the tag will respond with its potential next IDS. The reader uses the tag's response IDS to find a matched entry in the database, and goes to the mutual authentication phase if a matched entry is found; otherwise, it probes again and the tag responds with its old IDS. In the mutual authentication phase, the reader and the tag authenticate each other, and they respectively update their local pseudonym and the keys after successful authentication. After successful authentication, the tag stores the matched values to the entry $\left(I D S_{\text {old }}\left\|K 1_{\text {old }}\right\| K 2_{\text {old }}\right)$ and stores the updated values to the entry $\left(I D S_{\text {next }}\left\|K 1_{\text {next }}\right\| K 2_{\text {next }}\right.$ ). The random number generator is required on the reader only, and the tags only involve simple bit-wise operations like bitwise $\operatorname{XOR}(\oplus)$, bitwise OR $(\vee)$, bitwise AND $(\wedge)$, addition mod 
$2^{m}(+)$, and left rotate $(\operatorname{Rot}(x, y)) . \operatorname{Rot}(x, y)$ is defined to left rotate the value of $x$ with $y$ bits. The protocol procedures are described as follows.

Tag identification: Initially, the reader sends "hello" to the tag, which first responds with its potential next IDS. If the reader could find a matched entry in the database, it steps into the mutual authentication phase; otherwise, it probes again and the tag responds with its old IDS.

Mutual authentication phase: the reader uses IDS to find a matched record in the database. It could be the potential next IDS or the old IDS of the tag. It then uses the matched values and two generated random integers $n 1$ and $n 2$ to compute the values $A, B$, and $C$ (the calculation equations are specified in Fig. 9). From $A|| B|| C$, the tag first extracts $n 1$ from $A$, extracts $n 2$ from $B$, computes $\bar{K} 1$ and $\bar{K} 2$ and then verifies the value of $C$. If the verification succeeds, then it computes the response value $D$. Upon receiving $D$, the reader uses its local values to verify $D$.

Pseudonym updating and key updating: After the reader and the tag authenticated each other, they update their local pseudonym and keys as specified in Fig. 9. The scheme also provides confirmation of the synchronization values $(\bar{K} 1, \bar{K} 2)$ when the reader and the tag successfully authenticate each other.

\section{The weaknesses}

Sun et al. [62] had noticed that SASI is still vulnerable to DOS attacks. One attack scenario is described as follows. Assume that there is a synchronized tag $T_{x}$ in which $\left(I D S_{\text {next }}, K 1_{\text {next }}, K 2_{\text {next }}\right)$ equals to $\left(I D S_{1}, K 1_{1}, K 2_{1}\right)$ stored in the database. Now, suppose the reader probes the tag, and sends out $\left(A^{\prime}, B^{\prime}, C^{\prime}\right)$, which is eavesdropped by the attacker. At the end of the protocol, the attacker interrupts the message $D$ so that the reader will not update its variables. However, the tag will update its variables as follows: a) $\left.\left(I D S_{\text {old }}, K 1_{\text {old }}, K 2_{\text {old }}\right)=\left(I D S_{1}, K 1_{1}, K 2_{1}\right), \mathrm{b}\right)\left(I D S_{\text {next }}, K 1_{\text {next }}, K 2_{\text {next }}\right)=\left(I D S_{2}, K 1_{2}, K 2_{2}\right)$.

\begin{tabular}{|c|c|c|c|c|}
\hline & Reader & \multirow[b]{2}{*}{ 1. hello } & Tag & \\
\hline \multicolumn{2}{|c|}{ Tag identification: } & & \multicolumn{2}{|c|}{ The tag first responds with the next } \\
\hline \multicolumn{2}{|c|}{$\begin{array}{l}\text { Mutual authentication: } \\
\text { use IDS to find a matched recore }\end{array}$} & 2. IDS & \multirow{3}{*}{\multicolumn{2}{|c|}{$\begin{array}{l}I D S \text {, and then the old } I D S \text { if } \\
\text { necessary } \\
\text { Extract } n 1 \text { from } A \text {, and } n 2 \text { from } B \text {, }\end{array}$}} \\
\hline \multicolumn{2}{|c|}{$A=I D S \oplus K 1 \oplus n 1$} & 3. $A\|B\| C$ & & \\
\hline \multicolumn{2}{|c|}{$B=(I D S \vee K 2)+n 2$} & 4. $D$ & $\overline{-1}$ & \\
\hline \multicolumn{3}{|c|}{$\bar{K} 1=\operatorname{Rot}(K 1 \oplus n 2, K 1)$} & \multicolumn{2}{|c|}{$\bar{K} 1=\operatorname{Rot}(K 1 \oplus n 2, K 1)$} \\
\hline \multicolumn{3}{|c|}{$\bar{K} 2=\operatorname{Rot}(K 2 \oplus n 1, K 2)$} & \multicolumn{2}{|c|}{$\bar{K} 2=\operatorname{Rot}(K 2 \oplus n 1, K 2)$} \\
\hline \multicolumn{3}{|c|}{$C=(K 1 \oplus \bar{K} 2)+(\bar{K} 1 \oplus K 2)$} & \multicolumn{2}{|c|}{$\hat{C}=(K 1 \oplus \bar{K} 2)+(\bar{K} 1 \oplus K 2)$} \\
\hline \multicolumn{3}{|l|}{ Verify $D$} & \multicolumn{2}{|l|}{ verify $\hat{C}^{?}=C$} \\
\hline \multicolumn{5}{|c|}{ If $\mathrm{OK}$, } \\
\hline \multicolumn{3}{|c|}{ Pseudonym updating and key updating: } & \multicolumn{2}{|c|}{$D=(\bar{K} 2+I D) \oplus((K 1 \oplus K 2) \vee \bar{K} 1)$} \\
\hline \multicolumn{3}{|c|}{$I D S=(I D S+I D) \oplus(n 2 \oplus \bar{K} 1))$} & \\
\hline \multicolumn{2}{|c|}{$K 1=\bar{K} 1 ; \quad K 2=\bar{K} 2$} & & \multicolumn{2}{|l|}{$\begin{array}{l}I D S_{\text {odd }}=I D S ; I D S_{\text {mext }}=(I I \\
K 1_{\text {old }}=K 1 ; K 1_{\text {nett }}=\bar{K} 1 \\
K 2_{\text {old }}=K 2 ; K 2_{\text {mext }}=\bar{K} 2\end{array}$} \\
\hline
\end{tabular}

Fig. 9. SASI protocol 
Next, the attacker allows the reader and the tag to run the protocol again without intervening. Because $I D S_{2}$ is not found in the database, both the reader and the tag use $I D S_{1}$ to complete the authentication. Thus, the database will update its variable list to $\left(I D S_{3}, K 1_{3}, K 2_{3}\right)$, but the tag would own the values $\left(I D S_{1}, K 1_{1}, K 2_{1}\right)$ and $\left(I D S_{3}, K 1_{3}, K 2_{3}\right)$.

Finally, the attacker imitates as a valid reader to probe the tag. The tag first replies $I D S_{\text {next }}=I D S_{3}$, the attacker ignores this reply, which triggers the tag to reply $I D S_{1}$. The attacker now replays the recorded message $\left(A^{\prime}, B^{\prime}, C^{\prime}\right)$, which is valid and the tag would update its values a) $\left(I D S_{\text {old }}, K 1_{\text {old }}, K 2_{\text {old }}\right)=\left(I D S_{1}, K 1_{1}, K 2_{1}\right), \quad$ b) $\quad\left(I D S_{\text {next }}, K 1_{\text {next }}, K 2_{\text {next }}\right)$ $=\left(I D S_{2}, K 1_{2}, K 2_{2}\right)$. Now, the genuine reader and the tag are out-of-synchronization. Sun et al. had shown another attack scenario, and other researchers like [8, 35, 49] had further shown passive attack to disclose the secrets of tags.

\subsubsection{Multi-round authentication protocols- $\mathrm{HB}^{+}[27]$}

The HB protocol [20, 21], proposed by Hopper and Blum, is a multi-round protocol and is based on the hardness of the LPN (Learning parity with noise) problem. However, the HB protocol is only secure to passive attacks, and successive improvements like $[6,18,29,40,51]$ tried, but in vein, to protect from active attacks. In the following, we introduce the LPN problem and $\mathrm{HB}^{+}$protocol [27]. Interested readers are referred to [6, 18, 29, 40, 51] for other HB-related works.

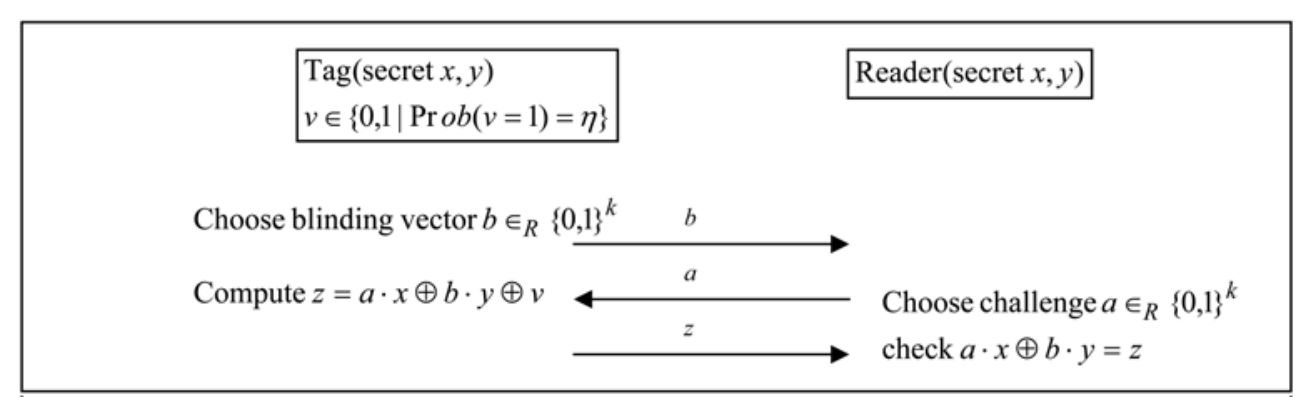

Fig. 10. One round of $\mathrm{HB}^{+}$

The LPN problem: The LPN problem with security parameters $q, k, \eta$, with $\eta \in\left[0, \frac{1}{2}\right]$ is defined as: given a random $q \times k$ binary matrix $A$, a random $k$-bit vector $x$, a vector $v$ such that $|v| \leq \eta q$, and the product $z=A \cdot x \oplus v$, find a $k$-bit vector $x^{\prime}$ such that $\left|A \cdot x^{\prime} \oplus z\right| \leq \eta q$.

$\mathbf{H B}^{+}$: Juels and Weis [27] tried to improve the HB protocol to resist active attacks. There are two $k$-bits secrets $x, y$ between the reader and the tag. The protocol is composed of $q$ rounds, one of which is depicted in Fig. 10. The tag is successfully authenticated if the check fails at most $q \eta$ times.

Gibert et al. [18] had shown a man-in-the-middle attack on $\mathrm{HB}^{+}$. In their model, they assume that an attacker can learn whether an authentication procedure succeeds or not. One attack scenario is depicted in Fig. 11. The attack consists of two phases. First, the attacker replaces the challenge $a$ sent by the reader with $a^{\prime}=a \oplus \delta$ in all $q$ rounds of the authentication 
process, where $\delta$ is a $k$-bit constant vector. If the authentication succeeds, she can conclude that $\delta \cdot x=0$ with high probability; otherwise, $\delta \cdot x=1$ with high probability. The attacker can set only one bit of $\delta$ on each time, and repeats the process $k$ times to reveal all the bits of $x$. In the second phase, the attacker impersonates the tag and sends a well chosen blinding vector $b$ to the reader. After that, she responds to the reader challenge $a$ with $z=x \cdot a$. If the authentication succeeds, she learns that $y \cdot b=0$ with high probability; otherwise, she concludes that $y \cdot b=1$ with high probability. After manipulating the bits of $b$ and repeating the process $k$ times, she can learn all the bits of $y$.

Even though several successive variants of $\mathrm{HB}^{+}$have been proposed [6, 9, 18, 29, 40, 51], none of them can resist all possible active attacks, and all the variants of $\mathrm{HB}$ series did not consider the anonymity and forward secrecy property.

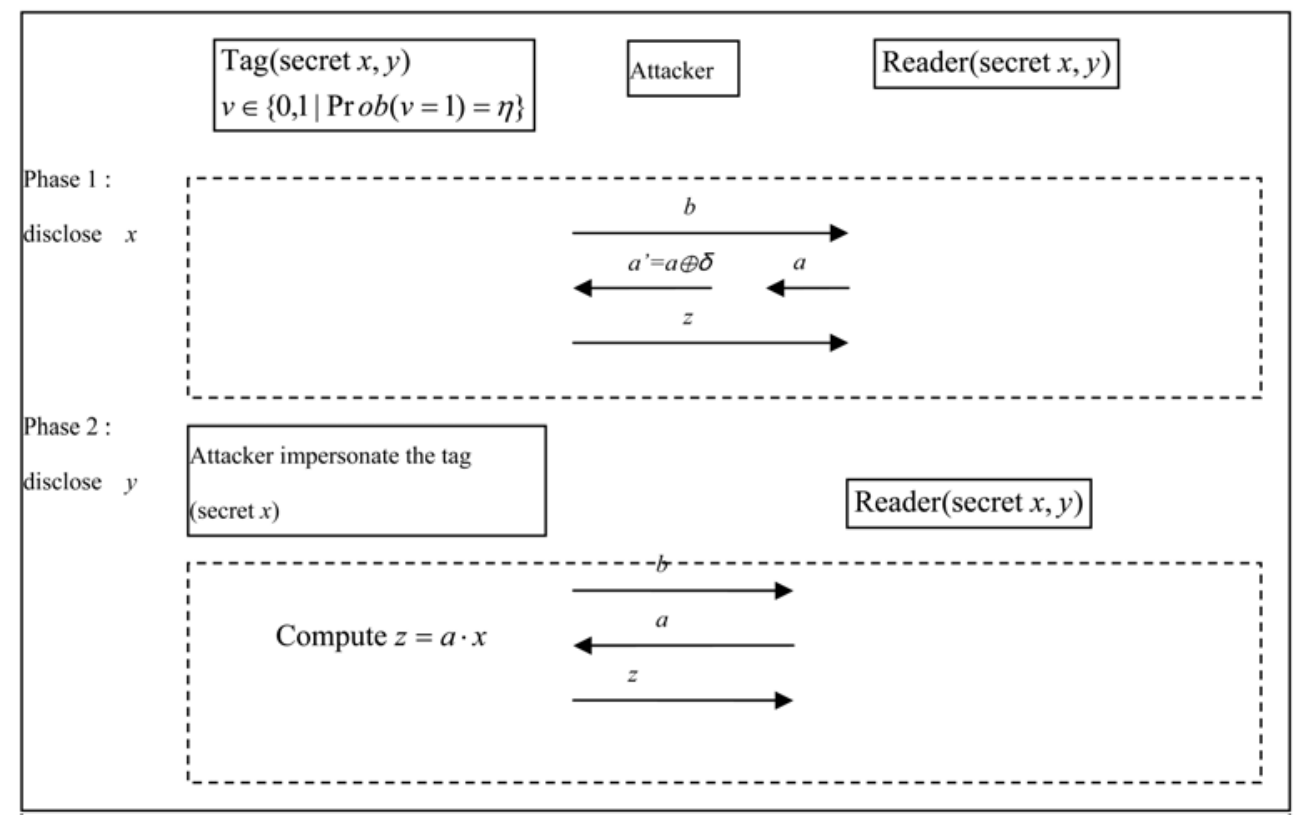

Fig. 11. Attack on $\mathrm{HB}^{+}$

\subsection{The resources-based classification of RFID authentication protocols}

In Section 2.1, we review and discuss several RFID authentication protocols without discussing their required resources. Actually, there are various RFID tags on the market, and the capacities of these tags are quite varying: some can support public key computations, and some can only support simple bit-wise operations. However, to have large market penetration, the cost of RFID tag plays an important factor, and most of the tags targeted for consumer market would be low-cost or even very low-cost. Even though most of the RFID authentication protocols introduced in Section 2.1 are targeted for such kind of tags, the required resources of these protocols are quite varying.

Based on the required capacity on tags, we roughly classify the RFID authentication protocols into four classes. The first class called "full-fledged class" refers to those protocols 
(like the schemes [25]) that demand the support of conventional cryptographic functions like symmetric encryption, cryptographic one-way function or even the public key algorithms. One of the main applications of these full-fledged protocols is E-passport and credit card. The second class called "simple" is for those protocols (like the schemes [10, 19, 38. 43, 59, 63, $64,66,67])$ that should support random number generator and one-way hashing function on tags

The third class called "lightweight" protocols refers to those protocols $[6,9,11,12,15,18,20$, $21,22,27-29,40,51]$ that require random number generator and simple functions like Cyclic Redundancy Code (CRC) checksum but not hash function. The EPC Gen 2 tag [17] supports both random number generator and CRC function.

The fourth class called "ultra-lightweight" refers to the protocols [8, 11, 13, 33-35, 45-47, 49, 62] that only involve simple bit-wise operations (like XOR, AND, OR, etc) on tags. PerisLopez et al. first proposed a series of ultra-lightweight authentication protocols [45-47], where the tags involve only simple bit-wise operations like XOR, AND, OR and addition mod $2^{m}$. These schemes are very efficient, and they only require about 300 gates. Unfortunately, LiWang [34] and Li-Deng [33] reported the de-synchronization attack and the full-disclosure attack on these protocols, and Chien and Hwang [13] further pointed out the weakness of Li-Wang's improved scheme. The security weaknesses of SASI protocols are explored in [8, $35,49,62]$. In addition to design ultra-light authentication protocols, other researcher like $[41,65]$ focused on designing lightweight hash function or encryption functions.

\subsection{The classification based on cryptographic approaches}

Contrary to the authentications in conventional applications where anonymity and untraceability are usually not necessary properties, anonymity and un-traceability are desirable properties in many RFID applications. Therefore, this section discusses those RFID authentication protocols that consider anonymity and un-traceability, and those protocols like $[6,18,29,40,51,63,64]$ that do not consider or do not well protect anonymity and untraceability are excluded from the following discussion. Based on the technique a RFID authentication protocol uses to identify a tag while protecting the anonymity, we may classify anonymous RFID authentication protocols into the following different approaches. In describing these approaches, we focus on the techniques to identify tags while preserving the anonymity, without covering the details of the protocols.

Simple challenge-response approach. In this approach, each tag $T_{i}$ shares a distinct key $k_{i}$ with the server $S /$ the reader $R$. When the reader $R$ probes a tag $T_{i}$ by sending a random value $N_{R}$ as a challenge, $T_{i}$ responds with $h\left(k_{i}, N_{R}\right)$, where $h()$ denotes a secure one-way function or some function that can output commitment on its inputs while protecting the un-disclosed input $k_{i}$. Upon receiving the response $h\left(k_{i}, N_{R}\right)$, the server computes $h\left(k_{j}, N_{R}\right)$ for each potential tag $T_{j}$ in its database to see whether there is a matched tag. This approach allows the server to identify a tag without disclosing the identity to eavesdroppers. Each tag just keeps one secret key, but the server needs to perform the computation for each potential tag to identify the tag. So, the tag's storage space is $\mathrm{O}(1)$ but the computational cost for identifying a tag is $\mathrm{O}(n)$, where $n$ the number of possible tags. The previous schemes like $[12,15,28,30,38,44,59,66,67]$ adopt this approach.

Tree-walk approach. In this approach, the tags are organized as a tree, where each leaf node in the tree denotes one tag and each edge in the tree is associated with a key. Fig. 12(b) shows 
one simple example. In the example, tag $T_{1}$ owns the key $K 1$ and $K 3$, and tag $T_{2}$ owns the keys $K 1$ and $K 4$. When a reader probes $T_{2}$ by sending a challenge $N_{R}, T_{2}$ responds with $\left\{h\left(K_{1}, N_{R}\right), \mathrm{s}\right\}$ on which the server can perform depth-first-search to identify the tag. This approach requires $\mathrm{O}(\log n)$ key space on each tag and demands $\mathrm{O}(\log n)$ computational cost to identify a tag. The key space requirement is a serious burden on low-cost tags. One more serious weakness of this approach is that once a tag is compromised, other tags that share the same keys on the same key paths could be partially traced. The more the number of keys one tag $T_{i}$ shares with the compromised tag $T_{j}$, the more the tag $T_{i}$ could be identified and traced. The schemes like [31, 32] adopt this approach.

Hash chains approach. One distinguished work of this approach is Ohkubo et al.'s scheme [43]. In this approach, the server and each tag $T_{x}$ shares a distinct hash seed $s_{1_{-} x}$ initially. $T_{x}$ updates $s_{i+1_{-} x}=h\left(s_{i_{-} x}\right)$ for $i \geq 1$ and responds with $a_{i_{-} x}=g\left(s_{i_{-} x}\right)$ for each query request, where $h() / g()$ are two different hash functions. This approach owns the forward secrecy property; that is, even assuming a tag is compromised one day in the future, the past communications from the same tag can not be traced. However, Ohkubo et al.'s original version cannot resist the replay attack [1], and has the poor scalability problem [2, 3] - the computational cost to identify a tag is $\mathrm{O}(\mathrm{nm})$, where $n$ is the number of potential tags and $m$ is the maximum length of the hash chain. Avoine et al. [1] discussed the techniques to conquer the replay attack, and Avoine et al. also [1,2] also proposed their improvements to reduce the time complexity at the cost of extra memory.

Varying Pseudonym (VP) approach. In this approach like [11, 19,45-47], each tag synchronizes its varying identifier and its internal state with the server. Please notice that, even though some challenge-response-based schemes like $[2,12,15]$ also synchronize the state between the tags and the server, these schemes do not send a varying pseudonym to facilitate the server perform fast identification; we, therefore, do not count them in this VP approach. The varying identifier is called pseudonym in [11, 45-47], and is called metaID in [19, 31, 32]. Here, we all refer to them the pseudonyms. Upon receiving a challenge request, a tag responds with the current pseudonym and the commitment on the challenge and the secret internal state. Based on the commitment, the server can verify the tag. During the authentication, the tag and the server respectively update their pseudonyms and their internal state. In this approach, the pseudonym not only protects the anonymity of the tag but also facilitates the server to identify the tag in its database with $\mathrm{O}(1)$ computational complexity, because the server can directly use the pseudonym to locate the corresponding entry in its database and perform necessary computations for this matched entry only. Further more, each tag only needs constant quantity of internal values- $\mathrm{O}(1)$ key storage. It is these excellent features that make it quite attractive than the other approaches. However, due to the synchronization requirement, the VP-based schemes are prone to the desynchronization attacks (or the denial of service attacks) [8, 13, 33-35, 49, 62], if adversaries can manipulate the communications such that the tag and the server are out of synchronization. Fig. 12 depicts the main ideas of these approaches.

\section{Security analysis of the mifare ultralight card and OV-chipkaart}

In Section 2, we have examined several RFID authentication protocols published in the literature. In this section and the next, we shall examine the security of some popular tags on 
the market. Section 3 will discuss the Mifare Ultralight card [36], and Section 4 will cover the EPC Class 1 Generation 2 card [16, 17].

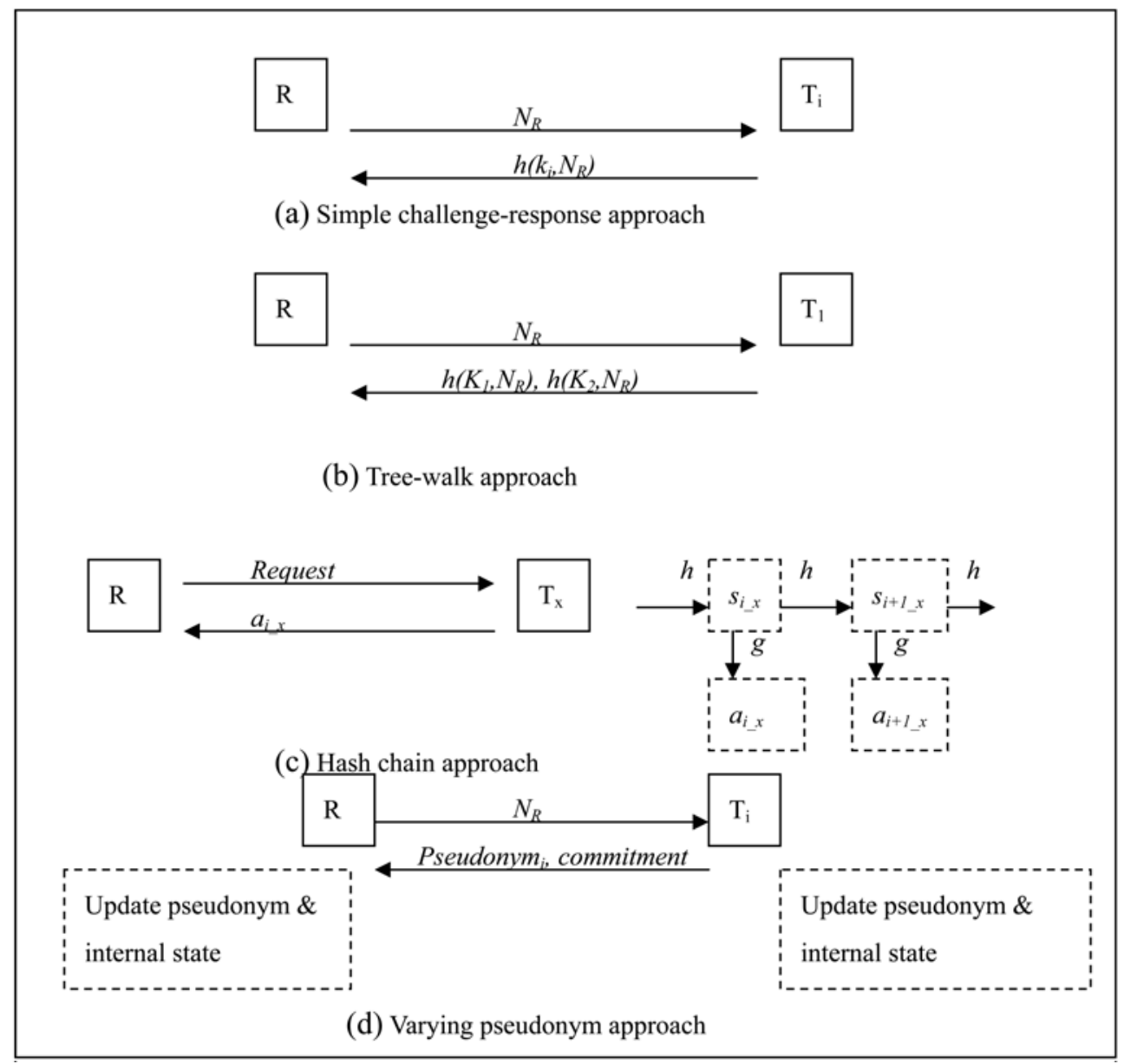

Fig. 12. Approaches to protect RFID tag identity

Mifare is a trademark of contactless RFID products and technologies developed by NXP Semiconductors [42]. Mifare cards have been widely used in many countries, and some of the cards feature a high level of security. However, Mifare Ultralight [42], one of Mifare card series, is focused on supporting faster applications at a cheaper cost. Thus, there is not any security mechanism implemented on the Mifare Ultralight chip. All privileges are fully accessible by anyone on the memory block. Section 3.1 right below will introduce Mifare card series, followed by Section 3.2 that deals with the memory organization of Mifare Ultralight. Then, Section 3.3 will take the OV-chipkaart in the Netherlands, which runs on the basis of the Mifare Ultralight card, as an example to discuss the security weaknesses and possible threats. 


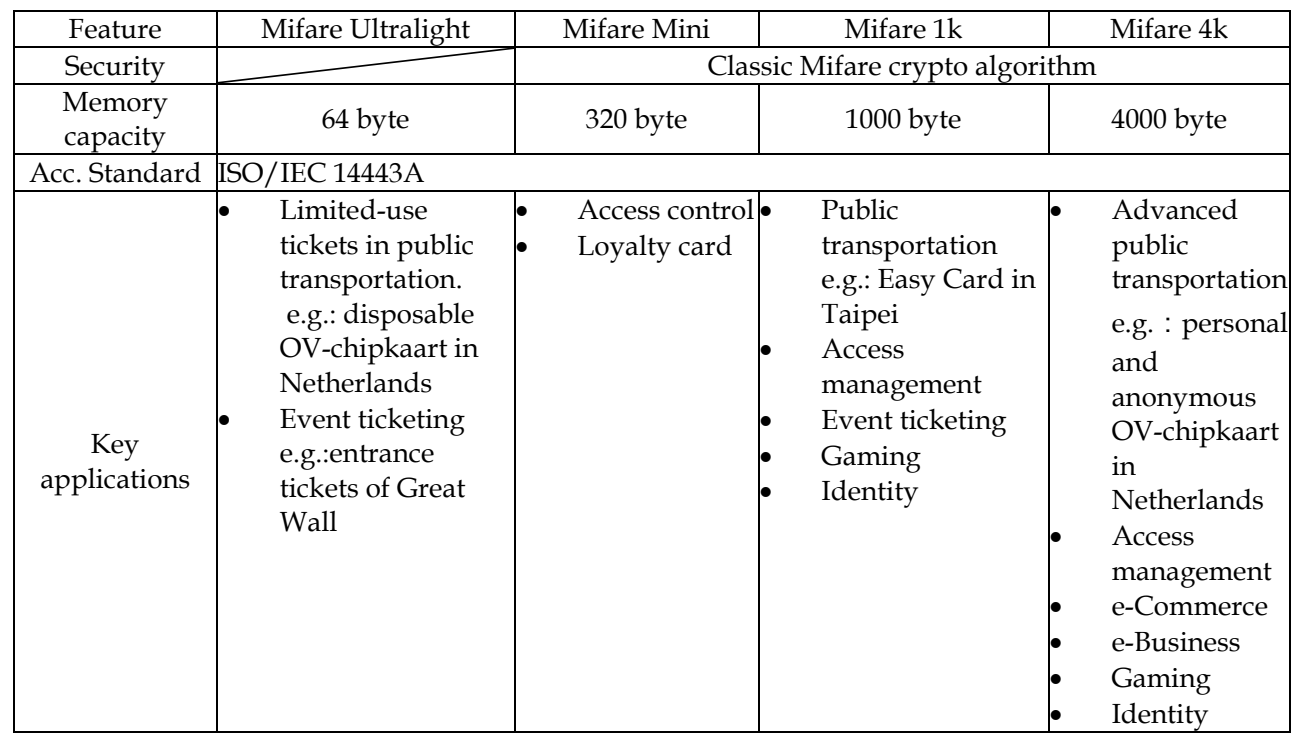

Table 1. Mifare series products' features and key applications (I) [36]

\subsection{Introduction}

Mifare is a trademark of contactless RFID products and technologies developed by a subsidiary company of Philips - the NXP Semiconductors [42]. With more than 1 billion smart card ICs and 5 million reader components sold around the world, Mifare is also the industry standard on the contactless RFID market, holding an $80 \%$ market share by value [36]. The applications of Mifare cards include automatic fare collection systems, inventory systems and household security systems, etc., and among them automatic fare collection systems are the most successful. Products of NXP Semiconductors include Mifare Ultralight, Mifare Mini, Mifare 1k, Mifare 4k, Mifare Plus, Mifare DESFire and Smart MX. Table 1 and Table 2 show the features and key applications of Mifare cards.

\begin{tabular}{|c|c|c|c|}
\hline Feature & Mifare Plus & Mifare DESFire & Smart MX \\
\hline Security & AES/DES & 3DES & 3DES/RSA/ECC \\
\hline Memory capacity & 2000/4000 byte & 4000 byte & $20000 \sim 144000$ byte \\
\hline Acc. Standard & \multicolumn{3}{|c|}{ ISO/IEC 14443A } \\
\hline Key applications & $\begin{array}{ll}\text { - } & \text { Public } \\
\text { transportation } \\
\text { - } & \text { Access } \\
\text { - } & \text { Evanagement } \\
\text { - } & \text { Gaming } \\
\text { - } & \text { Identity }\end{array}$ & 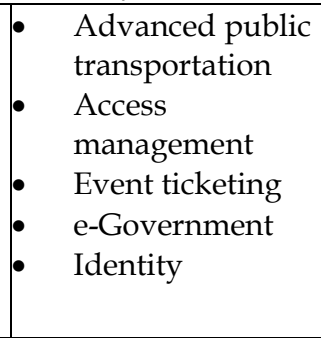 & $\begin{array}{ll} & \text { Advanced public } \\
\text { transportation } \\
\text { e.g. : e-Passport in } \\
\text { - } \text { Singapore } \\
\text { - } & \text { e-Government } \\
\text { - } & \text { Banking / Finance } \\
\text { Mobile } \\
\text { communication }\end{array}$ \\
\hline
\end{tabular}

Table 2. Mifare series products' features and key applications (II) [36] 
Mifare Ultralight, one popular card of Mifare series, is non-reloadable and usage-limited. It can be used as one way tickets, tourist weekend passes, zone-based tickets, and multiple trip tickets such as 10-ride tickets. Once the value recorded in the card IC has run out, the card becomes invalid and no further access is allowed. Therefore, it is a very good replacement for the traditional paper ticket. The Ultralight card can be easily integrated into the existing Mifare Classic applications without any additional cost and any additional equipment. The public transport payment system between Amsterdam and Rotterdam has used the OV-chipkaart [61] as access control tokens, and the disposable OV-chipkaart, one of the three types of OV-chipkaart, is a personal, anonymous, and as the name indicates, disposable ticket, and it is based on the Mifare Ultralight card design.

Mifare Ultralight IC [42] is a contactless RFID card which confirms to ISO/IEC14443A standard. The following list gives an overview of Mifare Ultralight:

- Operating distance: Up to $100 \mathrm{~mm}$ (depending on antenna geometry)

- Operating frequency: $13.56 \mathrm{MHz}$

- Fast data transfer: 106kbit/s

- No battery needed (energy is offered by reader)

- True anti-collision

- 7 byte serial number (Unique Identification, UID)

Each Mifare Ultralight card IC has its own UID. The anti-collision function can use the UID to distinguish a large number of cards in the communication field simultaneously, and then select one card per transaction. To support faster applications at cheaper costs, there is no security mechanism implemented in Mifare Ultralight; for instance, there is neither 3-way authentication support nor key storage on the IC chip.

\subsection{Mifare ultralight memory organization}

Mifare Ultralight contains an EEPROM of 512 bits, which are organized into 16 pages with 4 bytes each. The first 80 bits are reserved for manufacturer data and UID. The following 16 bits are used for the read-only locking mechanism, and the next 32 bits are the OTP area (One Time Programmable Area). The final 384 bits are user programmable read/write memory. Fig. 13 shows the Mifare Ultralight memory organization. In short, Mifare Ultralight divides the whole memory into four parts: (1) UID/serial number, (2) lock bytes, (3) OTP bytes and (4) data pages or user area.

UID/serial number: It contains a 7-byte UID, two check bytes and a 1 byte of internal data. It is stored in page 0 , page 1 and the first 2 bytes of page 2 (Fig. 13). These bytes are writeprotected after the IC manufacturer has programmed the card. The UID consists of the first three bytes of page 0 (SN0, SN1 and SN2) and the four bytes of page 1 (SN4, SN5, SN6 and SN7). Each card has a unique serial number so no collision happens. Each UID should be unique and should be unpredictable, and this way the UID can be used to prevent card forgery. The two check bytes, BCC0 and BCC1 defined as CT $\oplus$ SN0 $\oplus$ SN1 $\oplus$ SN2 and $\mathrm{SN} 3 \oplus \mathrm{SN} 4 \oplus \mathrm{SN} 5 \oplus \mathrm{SN} 6$ respectively, follow the regulation of ISO/IEC 14443-3. The CT field, which has a value of $0 \times 88$, is the cascade tag, and is used for compatibility with the Mifare Classic 4 byte UID. In addition, SN0 is reserved for the manufacturer ID, according to ISO/IEC 14443-3 and ISO/IEC 7816-6 AMD.1. 


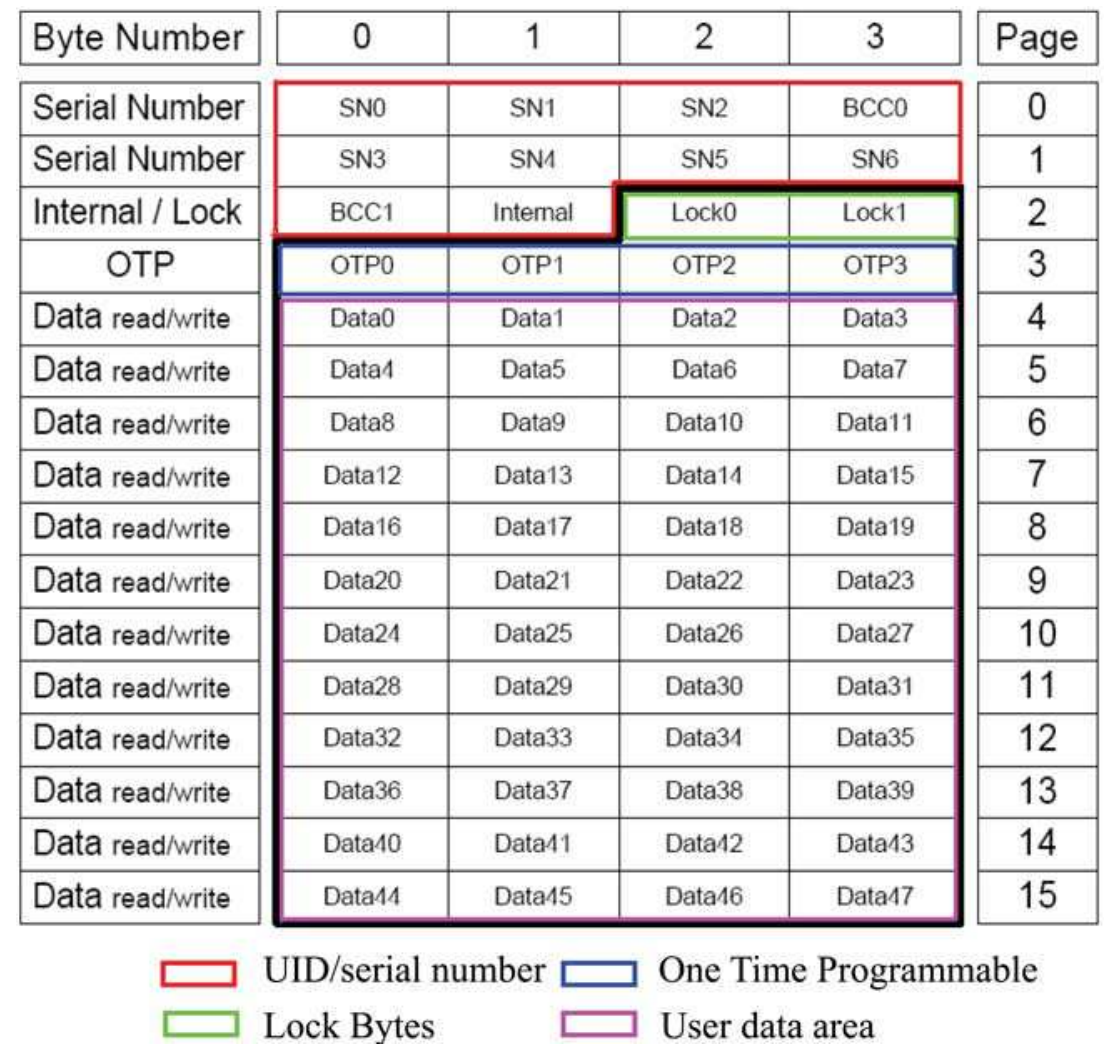

Fig. 13. Mifare Ultralight memory organization [42]

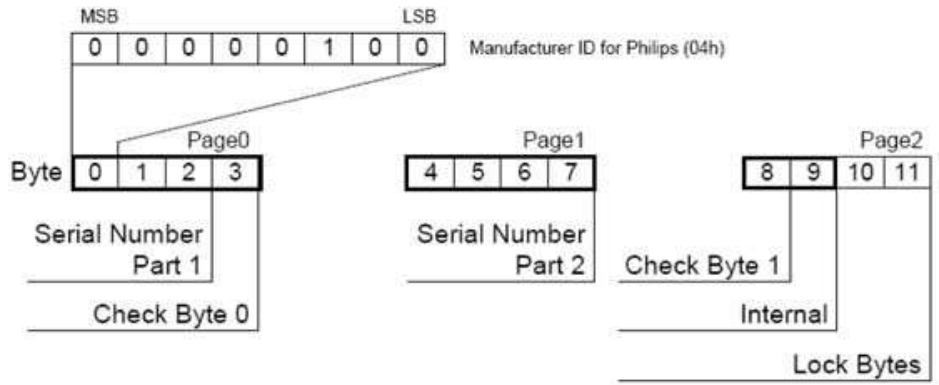

Fig. 14. Mifare Ultralight UID/Serial number[42]

(2) Lock Bytes: This part includes the last two bytes of page 2, which hold the fieldprogrammable read-only mechanism (see Fig. 15). The lock bytes include 3 block-locking bits (BL) and 13 locking bits (L). The initial value is set to " 0 ". The locking bits correspond to the pages from page 3 15 respectively, and when a locking bit is set to " 1 ," it indicates that the corresponding page is locked. The 3 block-locking bits of lock byte 0 on page 2 are the 
locks of locking bits, of which each bit handles pages 10 15, pages $4 \sim 9$ and page 3, respectively. Once the block-locking bit is set, the corresponding lock bits are locked. This process is irreversible; if a bit is set to" 1 ", then it cannot be altered to "0." For example, assume a block-locking bit, here let's take BL9-4 for example, is set to " 1 ", then the corresponding memory area L9 to L4 cannot be changed.

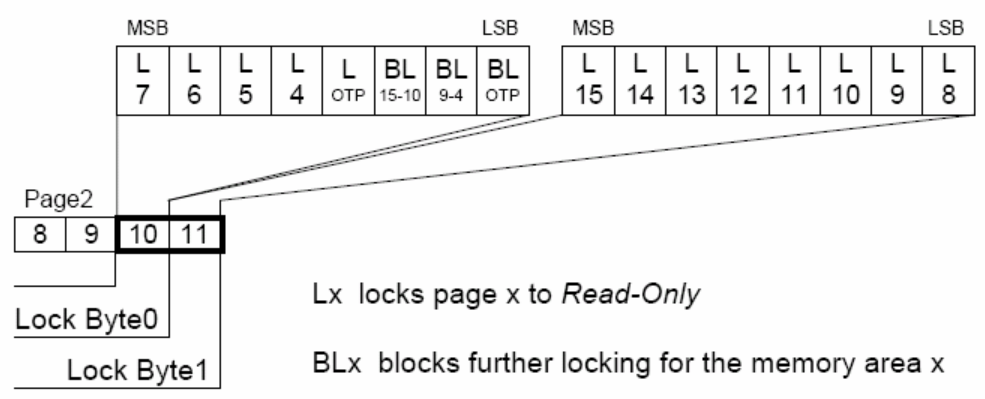

Fig. 15. Mifare Ultralight Lock Bytes [42]

(3) OTP Bytes: All the 32 bits of page 3 are the OTP bytes. The initial value of each bit is " 0 ", and it can only be set to " 1 " once, and the process is irreversible. The writing process of OTP byes is as follows. The current contents of the OTP bytes are bit-wise "or-ed" with the new bytes, and the result becomes the new contents of the OTP bytes (see Fig. 16). The OTP area can be used as a one-time counter with a maximum value of 32 (by setting every bit to 0 ). Please see Fig 17. For example, if a card has four rides available, then the value in the OTP bytes will be 1111111111111111111111111111 0000. An OTP with three rides available is 1111111111111111111111111111 0001. An OTP with no ride available is 111111111111 11111111111111111111.

\begin{tabular}{cc|c|c|}
\multicolumn{1}{c}{} & \multicolumn{3}{c}{ Page 3 } \\
\cline { 2 - 4 } Byte 12 & 13 & 14 & 15 \\
\hline & & \\
& & \\
& & & \\
& OTP Bytes \\
\hline
\end{tabular}

\begin{tabular}{|c|c|c|c|}
\hline \multicolumn{4}{|l|}{ Example } \\
\hline \multicolumn{2}{|c|}{ Default Value } & \multicolumn{2}{|c|}{ OTP Bytes } \\
\hline 00000000 & 00000000 & 00000000 & 00000000 \\
\hline \multicolumn{4}{|c|}{ 1st Write Command to page 3} \\
\hline 11111111 & 11111100 & 00000101 & 00000111 \\
\hline \multicolumn{4}{|c|}{ Result in page 3} \\
\hline 11111111 & 11111100 & 00000101 & 00000111 \\
\hline \multicolumn{4}{|c|}{ 2nd Write Command to page 3} \\
\hline 11111111 & 00000000 & 00111001 & 10000000 \\
\hline \multicolumn{4}{|c|}{ Result in page 3} \\
\hline 11111111 & 11111100 & 00111101 & 10000111 \\
\hline
\end{tabular}

Fig. 16. OTP Bytes and an example [42] 
(4) Data Pages: Pages 4 15 are user programmable read/write memory. These 384 bits are fully accessible to anyone. The values of these data pages are pre-set to " 0 ".

In the next section, we will examine the security of Netherlands' OV-chipkaart system, which is based on that of the Mifare Ultralight card. The following discussion is based on the report [61].

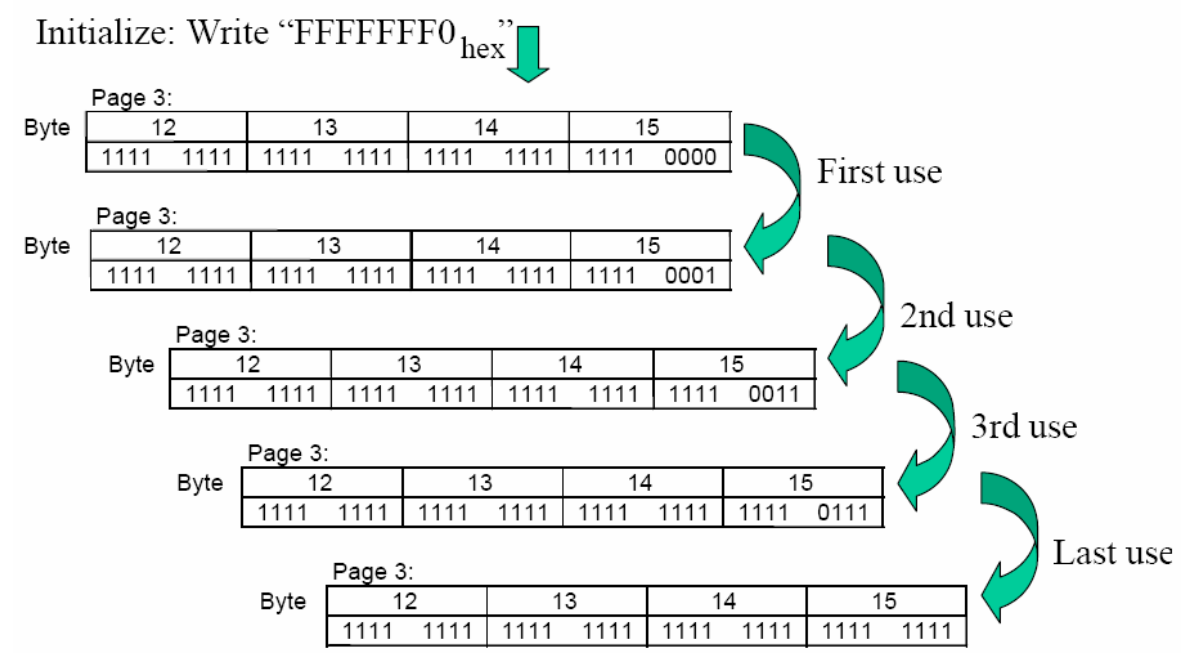

Fig. 17. Example-a four-ride ticket [42]

\subsection{The security weaknesses of OV-chipkaart on mifare ultralight}

The OV-chipkaart in use by the transport payment systems of Amsterdam and Rotterdam in the Netherlands is based on RFID technologies. The OV-chipkaart provides three types of tickets: personal tickets, anonymous tickets and disposable tickets. The personal ticket and the anonymous ticket are based on Mifare Classic $4 \mathrm{k}$, and the disposable ticket is based on Mifare Ultralight. Readers and Mifare Classic cards together can make the three-pass authentication process a reality, and then the transferred data get encrypted by using NXPdeveloping crypto algorithms. However, Mifare Ultralight neither provides three-pass authentication nor data encryption. The data on Mifare Ultralight are fully accessible to anyone, and the communication is not done under the protection of encryption. According to the report [61] released by University of Amsterdam, the disposable OV-chipkaart based on Mifare Ultralight has the three weaknesses as follows: (1) failure of defense mechanism, (2) repeated check-out and (3) free travel problem. In the discussion below, we shall focus on the Mifare-Ultralight-based OV-chipkaart, and refer to it as the OV-chipkaart for short.

In the OV-chipkaart, the data pages of the card are configured into three sectors of 4 pages each: the first sector (slot 1) consists of pages $4 \sim 7$, the second sector (slot 2) contains pages $8 \sim 11$, and the last sector consists of pages 12 15. The first two sectors are used to store the check-in and check-out transactions, and the last sector is used to store the product information such as the number of available rides and the expiry date. Data on the first two 
pages of slot 1 and slot 2 are stored in plain text while the last two pages are encrypted. The former records the transaction serial number, the type of transaction, the date and the time, and the latter contains the stations where the transactions took place.

There are three highlights in the lifetime of a disposable OV-chipkaart ticket: (1) getting purchased from a vending machine, (2) checking in when arriving at the station, and finally (3) checking out when leaving.

The legitimate access to the memory of a disposable OV-chipkaart ticket happens this way and in this order. (1) After a disposable ticket is purchased, a purchase transaction will be written into slot 2, and the lock bytes will be set as 0x00F0. Pages 12 15 are locked as readonly and cannot be changed anymore. (2) When arriving at a station, the user uses the disposable ticket to check-in. During the check-in process, the OTP bytes are set to indicate the number of remaining rides. The check-in transaction is written into slot 1 . If the value in the OTP bytes is $0 x F F F F$, the system will refuse the card since it has no more rides available. (3) When the traveler arrives at the destination station and checks out, a new check-out transaction is written into slot 2, and overwrites the purchase transaction. When the user uses the card again, a new check-in transaction is written into slot 1 , overwriting the old check-in transaction, and the new check-out transaction is written into slot 2 and overwrites the old check-out transaction. This process is repeated until no more rides are available.

To prevent an attacker from using an invalid card to check-in, the OV-chipkaart offers a defense mechanism. When someone attempts to use an invalid card to check-in, the defense mechanism gets triggered the moment the reader sets the lock bytes to 0xF8FF. Pages 3 15 enter a read-only state and no more access is allowed. Now this card is considered as permanently invalid.

The University-of-Amsterdam report [61] reveals that irregular usage behaviors disclose three vulnerabilities of OV-chipkaart: (1) failure of defense mechanism, (2) repeated checkout and (3) free travel. The report also proposes their countermeasures and improvements as follows.

(1.a) Failure of defense mechanism: Upon purchasing a disposable OV-chipkaart, the attacker sets all three block-locking bits to 1 to lock the locking bits from L3 through L15. Thus, when a reader detects an invalid card and fails to change locking bits, the defense mechanism fails.

(1.b) The solution: Adding a new checking function into the OV-chipkaart reader so if any card that is detected to have any block-locking bits set, that card is regarded as invalid and is refused.

(2.a) Check-out repetition problem: Because check-in transactions and check-out transactions are stored in user programmable read/write memory, one can readily reverse the state of transaction as follows: the attacker backs up the purchase transaction after purchasing a ticket, and then uses this back up data to overwrite the check-out transaction. This vulnerability would allow a bunch of people to use the same card to check out many times.

(2.b) The solution: In the OV-chipkaart system, the OTP counter is checked only for checkin, and is ignored when the ticket user checks out. Therefore, one proposed solution is to check the OTP for both check-in and check-out. Due to the feature of irreversibility of the OTP counter, the system can also keep record of the number of check-out transactions to prevent repeated check-out. 
(3.a) Free travel problem: Because the transactions are stored in user read/write memory, an attacker can back up pages $4 \sim 11$, follow normal check-in and check-out processes, and then write back the back-up data to the memory. This modified card can be used again and again until it expires.

(3.b) The solution: The weakness resulting in the free travel problem is that the system would not reject a modified card with manipulated pages $4 \sim 11$, even though the OPT counter shows that no more ride there is available. Therefore, the software for check-in and the OTP counter should both be redesigned carefully, getting every record of check-in and check-out transaction verified.

Because Mifare Ultralight neither supports authentication nor provides encryption, the security of the applications depends on the well-designed memory configuration and the software semantics. For example, one can adopt 3DES to encrypt the data before writing them back to the card, and can add the MAC (Message Authentication Code) to ensure the integrity of the stored data.

In addition to the weaknesses of Mifare Ultralight cards, the authors of [54, 55] have also found some forgery problems that might bug Mifare Classic cards. These reports further arouse people's attention on the security of RFIDs.

\section{Memory access and weaknesses of EPC Class1 Gen2}

EPC Class1 Generation 2 (Gen 2 for short) is one of the tag standards proposed by EPCglobal [16], which is believed to have great influence on the RFID consumer markets, especially the logistics management part. In a typical application, a reader first probes a Gen $2 \mathrm{tag}$, and then uses the information on the tag to further acquire the information of the labeled product. However, Gen 2 tags have limited computation capacity, and the communication protocols between readers and tags are not secure. These weaknesses result in privacy violation, tracking problems and cloning problems. This section discusses the memory configuration and the weaknesses of Gen 2 tags.

\subsection{Introduction}

During the 1990s, the Auto-ID Center at MIT [37] was established, and it soon developed the Electronic Product Code (EPC for short). Extended from the structure of barcode, EPC contains more information about an object than barcode. In Oct. 2003, Universal Code Council (UCC) and Electronic Article Numbering (EAN) purchased the technology of EPC from the Auto-ID Center. Then they founded a nonprofit organization EPCglobal [16] to advance the standards of EPC and EPC network and to enhance the efficiency of the supply chain operation.

EPC Class 1 Generation 2 (Gen 2 for short) [17], a passive tag proposed by EPCglobal, is designed to be applied universally in supply chains, and now it is ISO 18000-6C standard. Gen 2 tags use no battery, and they are powered by the electricity the readers provide. It communicates at UHF band-860 960MHz with the communication range stretching from $2 \mathrm{~m}$ to $10 \mathrm{~m}$. However, Gen 2 tags are easily interfered by metal or liquids, resulting in poor quality communication. Currently, Gen 2 tags are employed in a number of applications such as logistics management, luggage management, etc. In the future, the price of the Gen 2 tag will be around $\$ 0.05 \sim 0.1$ [58], and many related devices are being developed [57]. In the next sub-section, we shall introduce the memory organization of the Gen 2 tag. 


\subsection{Gen 2 tag memory configuration}

Gen 2 tag memory is logically divided into four banks: user memory (bank 11), TID memory (bank 10), EPC memory (bank 01) and reserved memory (bank 00). Each bank may have zero or more memory words ( 1 word $=16$ bits). Fig. 18 shows the Gen 2 tag logical memory.

- $\quad$ User memory (bank 11)

This bank allows users to organize and store user data.

- $\quad$ TID memory (bank 10)

This bank stores a unique tag ID which is 8 bits $\left(00_{\mathrm{h}} \sim 07_{\mathrm{h}}\right)$ of ISO/IEC 15963 class-identifier value - either $\mathrm{E} 0_{\mathrm{h}}$ or $\mathrm{E} 2 \mathrm{~h}$. If the class-identifier value is $\mathrm{E} 0_{\mathrm{h}}$, then the memory location $08_{\mathrm{h}} \sim 0 \mathrm{~F}_{\mathrm{h}}$ contains an 8-bit manufacturer identifier, and $10_{\mathrm{h}} \sim 3 \mathrm{~F}_{\mathrm{h}}$ contains a 48 -bit tag serial number which is assigned by the tag manufacturer.

- $\quad$ EPC memory (bank 01)

This bank consists of three parts: the Electronic Product Code (EPC), 16-bit Protocol-Control (PC) bits and a 16-bit cyclic redundancy code (CRC-16). The Electronic Product Code contains the header, general manager number, object class, and serial number. It is unique and can be extended to meet the requirements of different industries. Currently, the EPC can only be encoded either the 64-bit way or the 96-bit way, but the 256-bit method is on the way. Users, depending on the requirements, can choose their own way of encoding. The Protocol-Control (PC) bits record the lengths of PC and EPC, and the structure of EPC, whether it follows the EPCglobal ${ }^{\mathrm{TM}}$ Tag Data Standards or ISO/IEC 15961.

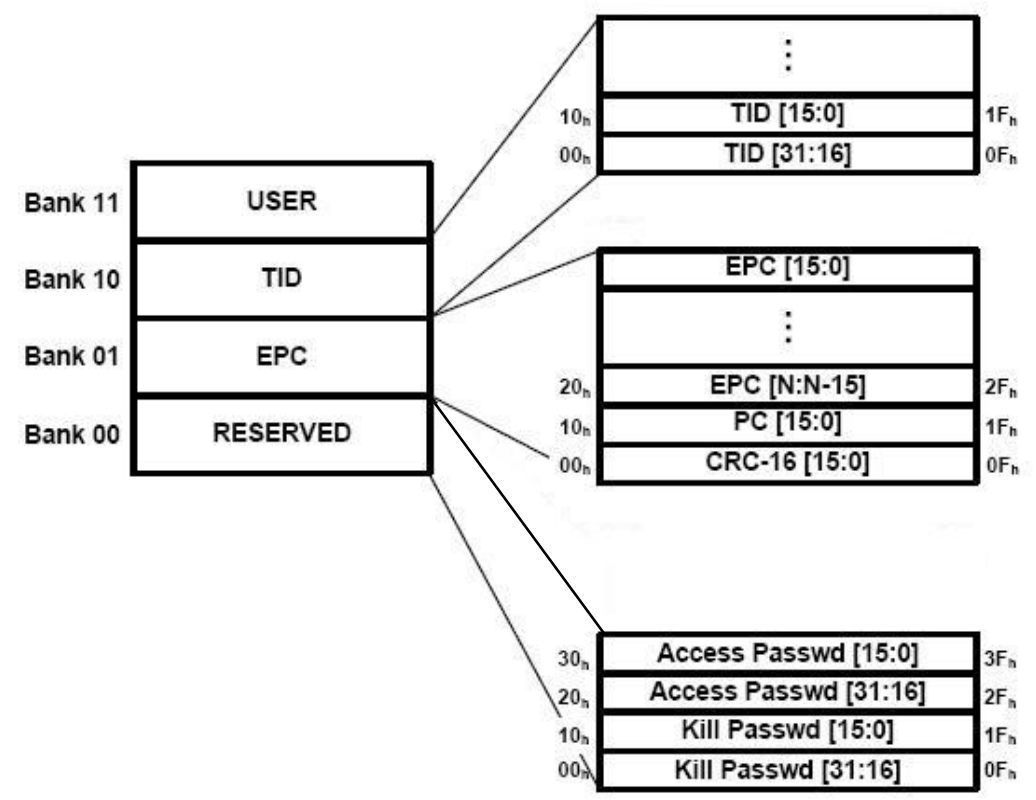

Fig. 18. Gen 2 tag logical memory [17]

- $\quad$ Reserved memory (bank 00)

This bank stores a 32-bit kill password and a 32-bit access password. 
- Kill password: A reader may pre-set a kill password to a tag. When the reader later issues the same kill password to the tag, the tag is permanently disabled. Once the reader executes the above operation, the tag is permanently silent. This function is used to disable the access to protect the privacy of the consumer when a product is purchased.

- Access password: A reader may pre-set an access password to a tag. When the reader later issues the same access password to the tag, the reader would lock one memory bank such as EPC memory, TID memory or User memory to prevent an un-authorized reader from writing data into the above-mentioned memory banks. Or the reader would lock a kill password or an access password to prevent an unauthorized reader from modifying the passwords.

The Gen 2 tag supports a 16-bit pseudo-random number generator (PRNG), a slot counter and the bitwise XOR operation. A 16-bit cyclic redundancy code (CRC) is used to protect the integrity of the communications. A 32-bit kill password is used to disable the access to protect the privacy of the consumer when a product is purchased, and a 32-bit access password is used to authenticate the readers so that no illegal writing will happen.

In the communications between readers and tags, each tag generates at least two random numbers-RN16 and handle. The tag uses the RN16 to identify whether a reader is the authenticated object, and the reader uses one or more handles to execute such operations as writing, locking, killing, etc. The communication protocols are to be introduced in the next section.

\subsection{Gen 2 communication protocols}

The communication protocols between readers and tags consist of three parts. Firstly, a reader picks a number of tags as the communication objects. Secondly, the reader obtains the EPC codes of the tags. After that, the reader will execute some specific operations such as reading, writing, locking, and killing. The process where the reader obtains the EPC code is shown in Fig. 19, and the steps are as follows.

Step 1. After the reader picks a number of tags, the reader issues a query command (which contains a 5-bit CRC) to start the communication between the reader and the tags.

Step 2. Upon receiving the query command, each of the tags generates a random number RN16 and computes a slot value. Then the reader issues some queryRep commands to the tags to make the tags' slot values to descend progressively. When the slot value of one of the tags hits zero, the tag responds to the reader with its RN16, and communicates with the reader.

Step 3. When receiving a random number $R N 16$, the reader sends the same $R N 16$ back to the tag as a reply. This way, the validity of the received RN16 can be checked, and the reader authenticated.

Step 4. If the verification succeeds, the tag transmits $E P C$ and $P C$ to the reader.

Step 5. After obtaining the tag's EPC, the reader executes operations such as reading, writing, locking and killing. Before executing the operations, the reader needs to issue a Req_RN command with the RN16 attached to it to make the tag respond with a new random number - the handle.

Step 6. The tag generates a new random number handle and responds to the reader with it.

Step 7. The reader executes such operations as reading, writing, locking and killing commands (the access commands in Fig. 19 that contain handle). If the reader executes 
a writing command to write data into the tag, the written data will be divided into successive words, and then each word is XORed with handle when it is transmitted to the tag. If the reader locks or kills the tag, the reader needs to input the access password or the kill password respectively. The password is divided into successive words, and then each word is XORed with handle when it is transmitted to the tag.

Step 8. The tag verifies the received handle. If the verification succeeds, the tag executes the specified operation (reading, writing, locking or killing), and then responds to the reader with handle. If the tag receives a reading operation, the tag responds with extra Data that the reader requests to read.

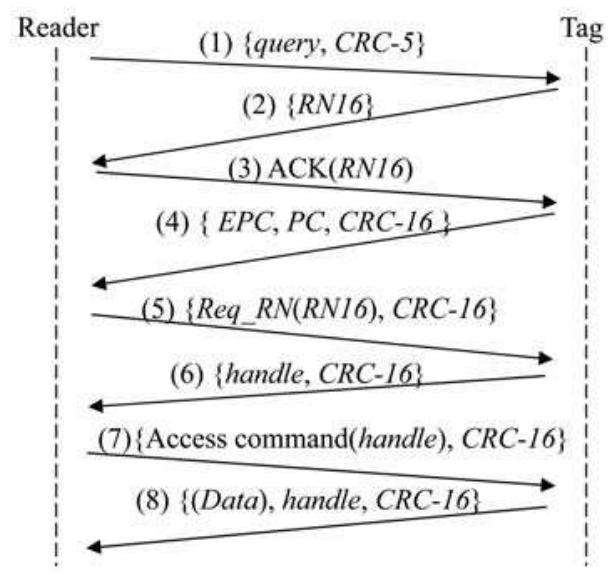

Fig. 19. The Gen 2 tag communication protocol

\subsection{The weaknesses}

Duc et al. [15] revealed that an attacker can easily derive the passwords from the communications because these passwords are only XORed with a transmitted random number. If an attacker obtains the random number handle in Step 6, then in Step 7 the tag's passwords could leak out, and in turn the attacker could access the contents in Step 8. Furthermore, please also notice that the Gen 2 tag always responds to any requests with its EPC code; therefore, it is not suitable for applications concerning anonymity or tracking. In this chapter, we have introduced RFID authentication protocols, have examined their security weaknesses, have classified these protocols into several categories and have discussed the features of these categories. We also introduce Mifare Ultra-light card and EPC gen 2, two popular tags on the market, and introduce the security of the two tags.

\section{Reference}

[1] G. Avoine, E. Dysli, and P. Oechslin, "Reducing time complexity in RFID systems," The 12th Annual Workshop on Selected Areas in Cryptography (SAC), LNCS 3897, pp. 291-306, Springer, 2006.

[2] G. Avoine, and P. Oechslin, "A scalable and provably secure hash-based RFID protocol," IEEE PerCom, 2005, pp. 110-114, 2005.

[3] G. Avoine, and P. Oechslin, "RFID traceability: a multi-layer problem," in Financial Cryptography 2005, LNCS 3570, pp. 125-140, Springer, 2005. 
[4] L. Bolotnyy, and G. Robins, "Generalized yoking-proofs for a group of RFID tags", in Proc. of MOBIQUITOUS 2006, July 2006.

[5] S. Brands and D. Chaum, "Distance-bounding protocols", In Advances in Cryptology EUROCRYPT'93, LNCS 765, pp. 344-359. Springer-Verlag, 1993.

[6] J. Bringer, H. Chabanne and E. Dottax, "HB++: A Lightweight Authentication Protocol Secure against Some Attacks," IEEE International Conference on Pervasive Service, Workshop on Security, Privacy and Trust in Pervasive and Ubiquitous Computing - SecPerU, 2006.

[7] M. Burmester, B. de Medeiros, and R. Motta, "Provably secure grouping-proof for RFID tags", Cryptology ePrint Archive, Report 2007/ 407, 2007.

[8] T. Cao, E. Bertino, H. Lei, "Security Analysis of the SASI Protocol", IEEE Transactions on Dependable and Secure Computing, 2008. http://doi.ieeecomputersociety.org /10.1109/TDSC.2008.32.

[9] H. Chabanne and G. Fumaroli, "Noisy Cryptographic Protocols for Low-Cost RFID Tags", IEEE Trans. on Information Theory 52(8), Aug. 2006.

[10] H. Y. Chien, "Secure Access Control Schemes for RFID Systems with Anonymity", accepted and to be printed in proceedings of 2006 International Workshop on Future Mobile and Ubiquitous Information Technologies (FMUIT'06), May, Japan.

[11] H. Y. Chien, "SASI: A New Ultra-Lightweight RFID Authentication Protocol Providing Strong Authentication and Strong Integrity," IEEE Transactions on Dependable and Secure Computing 4(4), October, 2007.

[12] H.-Y. Chien, and C.-H. Chen, "Mutual Authentication Protocol for RFID Conforming to EPC Class 1 Generation 2 Standards," Computers Standards \& Interfaces 29(2), pp 254-259, 2007.

[13] H.-Y. Chien, C.-W. Huang, "Security of Ultra-Lightweight RFID Authentication Protocols and Its Improvements," ACM Operating System Reviews 41(2), pp. 83-86, 2007.

[14] Y. Desmedt, "Major security problems with the'unforgeable' (Feige)-Fiat-Shamir proofs of identity and how to overcome them", in SecuriCom '88, pp. 15-17, SEDEP Paris, France, 1988.

[15] D. N. Duc, J. Park, H. Lee and K. Kim (2006), "Enhancing Security of EPCglobal Gen-2 RFID Tag against Traceability and Cloning," The 2006 Symposium on Cryptography and Information Security.

[16] EPCglobal, http://www.epcglobalinc.org/ .

[17] EPCglobal Inc., UHF Class 1 Gen 2 Standard Version 1.1.0, 2007, http://www.epcglobalinc.org/standards/uhfc1g2/ (2007/10/20).

[18] H. Gilbert, M. J.B. Robshaw, and Y. Seurin, "HB\#: Increasing the Security and E ciency of $\mathrm{HB}+"$ ", IACR eprint, eprint.iacr.org/2008/028.pdf.

[19] A. D. Henrici, and P. MÄuller (2004), "Hash-based Enhancement of Location Privacy for Radio-Frequency Identification Devices using Varying Identifiers," In the Proceedings of PerSec' 04 at IEEE PerCom, pp.149-153.

[20] N. Hopper and M. Blum. A Secure Human-Computer Authentication Scheme. Technical Re-port CMU-CS-00-139, Carnegie Mellon University, 2000.

[21] N. Hopper and M. Blum. Secure Human Identification Protocols. Adv. in Cryptology I Asia-crypt 2001, LNCS 2248, pp. 52-66, 2001.

[22] A. Juels (2005), "Strengthening EPC Tag against Cloning," To Appear in the Procceedings of WiSe '05.

[23] A. Juels, "Yoking proofs for RFID tag", in: Proc. of the Second IEEE Annual Conference on Pervasive Computing and Communications Workshops, pp. 138-142, DC, USA, 2004.

[24] A. Juels, "Generalized 'yoking-proofs' for a group of RFID tags", In MOBIQUITOUS 2006, 2006. 
[25] A. Juels, D. Molner, and D. Wagner, “Security and Privacy Issues in E-passports”, RSA Laboratories, and UC-Berkeley.

[26] A. Juels, R. Rivest, and M. Szydlo, “The Blocker Tag: Selective Blocking of RFID tags for Consumer Privacy," 8th ACM Conf. Computer and Comm. Security, V. Atluri, ed., ACM Press, 2003, pp. 103-111.

[27] A. Juels and S. Weis. Authenticating Pervasive Devices with Human Protocols. Adv. in Cryptology | Crypto 2005, LNCS vol. 3621, Springer-Verlag, pp. 293-308, 2005.

[28] S. Karthikeyan, M. Nesterenko (2005), "RFID security without extensive cryptography," Proceedings of the 3rd ACM workshop on Security of ad hoc and sensor networks, pp. 63-67.

[29] J. Katz and J.-S. Shin. Parallel and Concurrent Security of the HB and HB+ Protocols. Adv. in Cryptology - Eurocrypt 2006.

[30] J. Kim, D. Choi, I. Kim, and H. Kim, "Product authentication service of consumer's mobile RFID device," ISEC'06, pp. 1-6, 2006.

[31] S. M. Lee, Y. J. Hwang, D. H. Lee, and J. I. Lim, “Efficient Authentication for Low-Cost RFID Systems," International Conference on Computational Science and its Applications - ICCSA 2005, May 2005.

[32] Y. K. Lee and I. Verbauwhede, "Secure and Low-cost RFID Authentication Protocols," Adaptive Wireless Networks - AWiN, November 2005.

[33] T. Li, and R. H. Deng, "Vulnerability Analysis of EMAP-An Efficient RFID Mutual Authentication Protocol," The Second International Conference on Availability, Reliability and Security (AReS 2007), Vienna, 2007.

[34] T. Li, and G. Wang, "Security Analysis of Two Ultra-Lightweight RFID Authentication Protocols", IFIP SEC 2007, May 2007.

[35] T. Li, G. Wang, R. H. Deng, "Security Analysis on a Family of Ultra-lightweight RFID Authentication Protocols", Journal of Software 3(3), Mar. 2008.

[36] MifareNet, http://mifare.net/.

[37] MIT Auto-ID center. http://www.autoidcenter.org/.

[38] D. Molnar and D. Wagner (2004), "Privacy and security in library RFID: Issues, practices, and architectures," Conference on Computer and Communications Security CCS'04, pp. 210-219.

[39] J. Munilla and A. Peinado, “Distance bounding protocols for RFID enhanced by using void-challenges and analysis in noisy channel", Wireless Communications and Mobile Computing 2008, DOI:10.1002/wcm.590.

[40] J. Munilla, and A. Peinado, "HB-MP: a further step in the HB-family of lightweight authentication protocols," Computer Networks, doi:10.1016/ j.comnet.2007.01.011, 2007.

[41] NTRU. GenuID. http:/ / www.ntru.com/products/genuid.htm.

[42] NXP Semiconductors, "Mifare Ultralight features and hints“http://www.nxp.com/acrobat / other/identification/M073120.pdf.

[43] M. Ohkubo, K. Suzuki, and S. Kinoshita, "Cryptographic approach to 'Privacy-friendly' tag," in RFID Privacy workshop, MIT, USA, 2003.

[44] K. Osaka, T. Takagi, K. Yamazaki, and O. Takahashi, "An efficient and secure RFID security method with ownership transfer," Proc. of International Conference on Computational Intelligence and Security 2006, pp. 1090-1095, LNCS 9743, Springer, 2006.

[45] P. Peris-Lopez, J. C. Hernandez-Castro, J. M. Estevez-Tapiador, and A. Ribagorda, “ LMAP: A Real Lightweight Mutual Authentication Protocol for Low-cost RFID tags", in: Proc. of 2nd Workshop on RFID Security, July 2006.

[46] P. Peris-Lopez, J. C. Hernandez-Castro, J. M. Estevez-Tapiador, and A. Ribagorda, "M2AP: A Minimalist Mutual-Authentication Protocol for Low-cost RFID Tags", in: Proc. of International Conference on Ubiquitous Intelligence and Computing UIC'06, LNCS 4159, pp. 912-923, Springer, 2006. 
[47] P. Peris-Lopez, J. C. Hernandez-Castro, J. M. Estevez- Tapiador, and A. Ribagorda, "EMAP: An Efficient Mutual Authentication Protocol for Low-cost RFID Tags," in: OTM Federated Conferences and Workshop: IS Workshop, November 2006.

[48] P. Peris Lopes, J. Hernandez-Castro, J.M. Estevez-Tapiador, and A. Ribagorda, "Solving the Simultaneous Scanning problem Anonymously: Clumping proofs for RFID Tags", in Proc. of SecPerU'07, 2007.

[49] R. C.-W. Phan, "Cryptanalysis of a New Ultralightweight RFID Authentication Protocol - SASI", IEEE Transactions on Dependable and Secure Computing, 19 June 2008. http://doi.ieeecomputersociety.org/10.1109/ TDSC. 2008.33.

[50] T. Phillips, T. Karygiannis, R. Kuhn (2005), "Security Standards for the RFID Market," IEEE Security \& Privacy, Vol. 3, No. 6, pp. 85-89.

[51] S. Piramuthu, "HB and Related Lightweight Authentication Protocols for Secure RFID Tag/Reader Authentication," CollECTeR Europe Conference, June 2006.

[52] S. Piramuthu, "Protocols for RFID tag/reader authentication", Decision Support Systems 43(3), pp. 897-914, 2007.

[53] S. Piramuthu, "On existence proofs for multiple RFID tags", in: Proc. of IEEE International Conference on Pervasive Services, Workshop on Security, Privacy and Trust in Pervasive and Ubiquitous Computing- SecPerU 2006, France, June 2006. IEEE.

[54] H. Plötz and K. Nohl, " Mifare little security, despite obscurity", 24th Chaos Communication Congress, http://events.ccc.de/congress/2007/Fahrplan/ events/2378.en.html.

[55] The Digital Security research group of the Radboud University Nijmegen, "Security Flaw in Mifare Classic", http://www.ru.nl/ds/research/rfid/.

[56] J. Reid, J.M. Gonzlez, T. Tang, B. Senadji, “Detecting Relay Attacks with Timing-Based Protocols. http:/ / eprints.qut.edu.au/archive/00003264/.

[57] RFID Journal, http://www.rfidjournal.com/.

[58] RFID Journal. Gillette to Purchase 500 Million EPC Tags. http://www.rfidjournal.com, January 2003.

[59] K. Rhee, J. Kwak, S. Kim, and D. Won (2005), “Challenge-response based RFID authentication protocol for distributed database environment," International Conference on Security in Pervasive Computing - SPC 2005, pp. 70-84.

[60] J. Saito and K. Sakurai, "Grouping proof for RFID tags", in: Proc, of AINA 2005, Taiwan, March 2005. IEEE.

[61] P. Siekerman, M. v. d. Schee, "Security Evaluation of the disposable OV-chipkaart", System and Network Engineering, University of Amsterdam, http:/ / staff.science.uva.nl / delaat/sne-2006-2007/p41/Report.pdf

[62] H. M. Sun, W. C. Ting, and K. H. Wang, "On the security of Chien's Ultra-lightweight RFID authentication protocol", IACR eprint, eprint.iacr.org/2008/083.pdf.

[63] S. A. Weis, (2003), "Security and Privacy in Radio-Frequency Identification Devices," Masters Thesis MIT.

[64] S. A. Weis, S. E. Sarma, R. L. Rivest, and D. W. Engels, "Security and Privacy Aspects of Low-Cost Radio Frequency Identification Systems," In the Proceedings of the First Security in Pervasive Computing, LNCS, Vol. 2802, pp.201-212, 2003.

[65] D. J. Wheeler and R. M. Needham, "TEA, a tiny encryption algorithm", technical report, Computer Laboratory, University of Cambridge, 1995.

[66] J. Yang, J. Park, H. Lee, K. Ren, and K. Kim. (2005), “Mutual authentication protocol for low-cost RFID," Handout of the Ecrypt Workshop on RFID and Lightweight Crypto.

[67] J. Yang, K. Ren and K. Kim (2005), "Security and privacy on authentication protocol for low-cost radio," The 2005 Symposium on Cryptography and Information Security. 


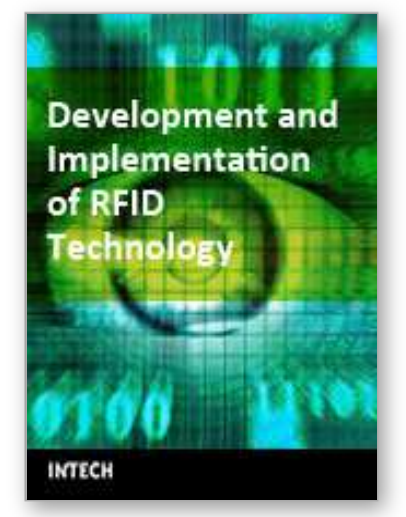

\author{
Development and Implementation of RFID Technology \\ Edited by Cristina Turcu
}

ISBN 978-3-902613-54-7

Hard cover, 450 pages

Publisher I-Tech Education and Publishing

Published online 01, January, 2009

Published in print edition January, 2009

The book generously covers a wide range of aspects and issues related to RFID systems, namely the design of RFID antennas, RFID readers and the variety of tags (e.g. UHF tags for sensing applications, surface acoustic wave RFID tags, smart RFID tags), complex RFID systems, security and privacy issues in RFID applications, as well as the selection of encryption algorithms. The book offers new insights, solutions and ideas for the design of efficient RFID architectures and applications. While not pretending to be comprehensive, its wide coverage may be appropriate not only for RFID novices but also for experienced technical professionals and RFID aficionados.

\title{
How to reference
}

In order to correctly reference this scholarly work, feel free to copy and paste the following:

Hung-Yu Chien (2009). The Study of RFID Authentication Protocols and Security of Some Popular RFID Tags, Development and Implementation of RFID Technology, Cristina Turcu (Ed.), ISBN: 978-3-902613-54-7, InTech, Available from:

http://www.intechopen.com/books/development_and_implementation_of_rfid_technology/the_study_of_rfid_au thentication_protocols_and_security_of_some_popular_rfid_tags

\section{INTECH}

open science | open minds

\section{InTech Europe}

University Campus STeP Ri

Slavka Krautzeka 83/A

51000 Rijeka, Croatia

Phone: +385 (51) 770447

Fax: +385 (51) 686166

www.intechopen.com

\section{InTech China}

Unit 405, Office Block, Hotel Equatorial Shanghai

No.65, Yan An Road (West), Shanghai, 200040, China

中国上海市延安西路65号上海国际贵都大饭店办公楼 405 单元

Phone: +86-21-62489820

Fax: $+86-21-62489821$ 
(C) 2009 The Author(s). Licensee IntechOpen. This chapter is distributed under the terms of the Creative Commons Attribution-NonCommercialShareAlike-3.0 License, which permits use, distribution and reproduction for non-commercial purposes, provided the original is properly cited and derivative works building on this content are distributed under the same license. 